\title{
THE NORTH AMERICAN AND PELICAN NEBULAE. I. IRAC OBSERVATIONS
}

\author{
S. Guieu ${ }^{1}$, L. M. Rebull ${ }^{1}$, J. R. Stauffer ${ }^{1}$, L. A. Hillenbrand ${ }^{2}$, J. M. Carpenter ${ }^{2}$, A. Noriega-Crespo ${ }^{1}$, D. L. Padgett ${ }^{1}$, \\ D. M. Cole ${ }^{3}$, S. J. Carey ${ }^{1}$, K. R. Stapelfeldt ${ }^{3}$, and S. E. Strom ${ }^{4}$ \\ ${ }^{1}$ Spitzer Science Center/Caltech, M/S 220-6, 1200 E. California Blvd., Pasadena, CA 91125, USA; guieu@ipac.caltech.edu \\ ${ }^{2}$ Department of Astronomy, California Institute of Technology, Pasadena, CA 91125, USA \\ 3 Jet Propulsion Laboratory, MS 183-900, California Institute of Technology, Pasadena, CA 91109, USA \\ ${ }^{4}$ NOAO, Tucson, AZ, USA \\ Received 2008 November 25; accepted 2009 March 4; published 2009 May 5
}

\begin{abstract}
We present a $9 \mathrm{deg}^{2}$ map of the North American and Pelican Nebulae regions obtained in all four Infrared Array Camera (IRAC) channels with the Spitzer Space Telescope. The resulting photometry is merged with that at $J H K_{s}$ from Two Micron All Sky Survey and a more spatially limited BVI survey from previous ground-based work. We use a mixture of color-color diagrams to select a minimally contaminated set of more than 1600 objects that we claim are young stellar objects (YSOs) associated with the star-forming region. Because our selection technique uses infrared excess as a requirement, our sample is strongly biased against inclusion of Class III YSOs. The distribution of IRAC spectral slopes for our YSOs indicates that most of these objects are Class II, with a peak toward steeper spectral slopes but a substantial contribution from a tail of Flat spectrum and Class I type objects. By studying the small fraction of the sample that is optically visible, we infer a typical age of a few Myr for the low-mass population. The young stars are clustered, with about a third of them located in eight clusters that are located within or near the LDN 935 dark cloud. Half of the YSOs are located in regions with surface densities higher than $1000 \mathrm{YSOs} / \mathrm{deg}^{2}$. The Class I objects are more clustered than the Class II stars.
\end{abstract}

Key words: circumstellar matter - infrared: ISM - infrared: stars - ISM: clouds - ISM: individual (NGC 7000, IC 5070) - stars: formation - stars: pre-main sequence

Online-only material: color figures, machine-readable table

\section{INTRODUCTION}

Much of our current knowledge regarding star-forming patterns and circumstellar disk evolution derives from the study of molecular cloud complexes within a few hundred parsecs of the Sun. Among these are a large number of lower mass clouds such as Taurus and, more infrequently, dense clouds like Orion, which is the prototypical high-mass and high-density star-forming region. While nearby cloud complexes serve as our primary empirical guide to understanding the formation and early evolution of stars, it is important that we study more than just the nearest examples. One little-studied-to-date star-forming region beyond the solar neighborhood is the North American and Pelican Nebulae (NGC 7000 and IC 5070, respectively), toward $l=85$, $b=-0.5$, about $600 \mathrm{pc}$ from the Earth. This region is probably more representative of star formation in the disk of the Milky Way than Taurus or Orion, it is also the next closest region after Orion with a substantial numbers of intermediate- and highmass stars. The physical appearance of the nebulae on optical images is thought to reflect a combination of a large, background H II region (W80) overlaid by several foreground, dark clouds, with the edges of the dark clouds illuminated by the optically unseen primary exciting star of the $\mathrm{H}$ II region. Because these nebulae lie essentially in the plane of the Galaxy and, as seen by us, along a spiral arm, study of the region is further confused by the juxtaposition of young stars and star-forming regions at a variety of distances. For the purposes of the rest of the paper, we will refer to the entire region as the NANeb, or the NAN complex.

Herbig (1958) was one of the first to study the star formation associated with these nebulae. He identified $68 \mathrm{H} \alpha$ emissionline stars from objective-prism plates of the region, including a small cluster of pre-main-sequence (PMS) stars in the "Gulf of Mexico" portion of the NANeb. Many of the young stars identified by Herbig lie along the bright rims of the dark clouds, possibly indicating that they are recent, second generation star formation products triggered by the expanding $\mathrm{H}$ II region from the first generation of stars in the NANeb. Herbig estimated a rough distance of $500 \mathrm{pc}$ for the star-forming complex, but with a variety of caveats related to whether this distance applied to some or all of the stars and nebulae. The best available modern distance for the region appears to be that of Laugalys \& Straižys (2002), who estimate a distance of $\simeq 600 \mathrm{pc}$. We will use this distance for the purposes of the paper with the caveats noted above.

Bally \& Scoville (1980) mapped the CO associated with the dark clouds that define the Gulf of Mexico and Atlantic Ocean, and estimated that the mass in gas still present is of the order of $3 \times 10^{4} M_{\odot}$. Their interpretation of the CO data was that the remaining dark clouds are the remnants of a much larger molecular cloud complex that has largely been disrupted by the hot, massive stars that were formed a few million years ago. Cambrésy et al. (2002) used the Two Micron All Sky Survey (2MASS; Skrutskie et al. 2006) point-source catalog to map the extinction and star clustering toward this region, deriving extinctions of $A_{v} \geqslant 35$ magnitudes in some portions of the darkest cloud (Lynds 935). Cambrésy et al. also identified nine apparent star clusters in the 2MASS data.

Bally \& Reipurth (2003) obtained narrow-band $\mathrm{H} \alpha$ and [S II] imaging of the region of the Pelican nebula, and identified a number of new $\mathrm{HH}$ objects and collimated outflows, mostly originating from the interface region between the dark cloud and the $\mathrm{H}_{\mathrm{II}}$ region. In a couple of cases, the flows originate from near the tip of elephant trunks. The exciting sources for 
most of the flows could not be identified from the existing data. Armond et al. (2003) obtained additional narrow- and broadband optical and near-infrared (near-IR) imaging of the NANeb, and identified 28 more $\mathrm{HH}$ objects, including many associated with the PMS stars of Herbig's Gulf of Mexico cluster. More recently, the probable exciting source for the $\mathrm{H}$ II region has been identified as an $\mathrm{O} 5$ star behind about $A_{V}=10$ mag of extinction, located inside the dark cloud LDN 935 separating the North America and the Pelican Nebulae (Comerón \& Pasquali 2005).

We have conducted a large ( $\left.\sim 9 \mathrm{deg}^{2}\right)$ IR imaging survey with the Spitzer Space Telescope (Werner et al. 2004) of this region, along with supporting data obtained in the optical for the $\sim 2.4 \times 1.7$ central region. This present paper, the first of a series, presents the Infrared Array Camera (IRAC) data. Future papers will cover the Multiband Imaging Photometer for Spitzer (MIPS) data, with special emphasis on the Gulf of Mexico cluster (L. M. Rebull et al. 2009, in preparation, hereafter R09), and the optical classification spectroscopy (L. A. Hillenbrand et al. 2009, in preparation).

First, we present the observational details for the IRAC observations (Section 2). We then use the IRAC colors to select a minimally contaminated, if not complete, sample of young stellar object (YSO) candidates (Section 3), and discuss their properties (classes, spatial distribution) in the context of other star-forming regions. Because IRAC is very sensitive to emission from $\mathrm{HH}$ objects, we then discuss the IRAC observations of one of the $\mathrm{HH}$ objects found in this region (Section 4).

\section{OBSERVATIONS, ANCILLARY DATA, AND BASIC DATA REDUCTION}

\subsection{Observations}

The Spitzer observations of the NAN complex were obtained as part of the Cycle-2 program 20015 (PI: L. Rebull). Additional observations of the "corners" of the map were obtained as part of program 462 in an effort to increase the legacy value of the data set. The IRAC observations from program 20015 were obtained on 2006 August 9-11, and the IRAC observations from program 462 were obtained on 2007 November 15-27. IRAC (Fazio et al. 2004) observes at 3.6, 4.5, 5.8, and $8 \mu \mathrm{m}$.

The Cycle-2 IRAC observations were designed to cover the region of highest extinction in a manner as independent of observing constraints as possible. Figure 1 is the NANeb region in the optical (from the Digitized Palomar Optical Sky Survey). The region covered by our IRAC map is indicated, as is the $A_{v}=5$ contour from the Cambrésy et al. (2002) extinction map. The observations were broken into 12 astronomical observation requests (AORs); the AORKEYs are given in Table 1. This region is at a high ecliptic latitude $\left(+57^{\circ}\right)$, so the field of view (FOV) rotates by about a degree a day, necessitating small individual AOR coverage in order to completely tile the region without leaving gaps. Also, because of the high ecliptic latitude, asteroids are not a significant concern, and we therefore obtained all of the imaging at a single epoch. Each mapping AOR was constructed with the same strategyat each map step, four dither positions were observed, each with high dynamic range (HDR) exposures of 0.6 and $12.0 \mathrm{~s}$ frame times. For this strategy, the on-line SENS-PET for a high background region reports $3 \sigma$ point-source sensitivities of 7.2, $10.7,66$, and $78 \mu \mathrm{Jy}$ for the four IRAC bands, respectively. Differential source count histograms for our point sources are
Table 1

Summary of IRAC Observations (Programs 20015 and 462)

\begin{tabular}{|c|c|c|}
\hline Program : Field ID & Map Center & AORKEY \\
\hline P20015 : 12 & $20 \mathrm{~h} 55 \mathrm{~m} 30.00 \mathrm{~s},+44 \mathrm{~d} 59 \mathrm{~m} 00.0 \mathrm{~s}$ & 16790528 \\
\hline P20015 : 13 & $20 \mathrm{~h} 53 \mathrm{~m} 06.00 \mathrm{~s},+44 \mathrm{~d} 59 \mathrm{~m} 00.0 \mathrm{~s}$ & 16790784 \\
\hline P20015 : 21 & $20 \mathrm{~h} 57 \mathrm{~m} 54.00 \mathrm{~s},+44 \mathrm{~d} 23 \mathrm{~m} 00.0 \mathrm{~s}$ & 16791040 \\
\hline P20015 : 22 & $20 \mathrm{~h} 55 \mathrm{~m} 30.00 \mathrm{~s},+44 \mathrm{~d} 23 \mathrm{~m} 00.0 \mathrm{~s}$ & 16791296 \\
\hline P20015 : 23 & $20 \mathrm{~h} 53 \mathrm{~m} 06.00 \mathrm{~s},+44 \mathrm{~d} 23 \mathrm{~m} 00.0 \mathrm{~s}$ & 16791552 \\
\hline P20015 : 24 & $20 \mathrm{~h} 50 \mathrm{~m} 42.00 \mathrm{~s},+44 \mathrm{~d} 23 \mathrm{~m} 00.0 \mathrm{~s}$ & 16791808 \\
\hline P20015 : 31 & $20 \mathrm{~h} 57 \mathrm{~m} 54.00 \mathrm{~s},+43 \mathrm{~d} 47 \mathrm{~m} 00.0 \mathrm{~s}$ & 16792064 \\
\hline P20015 : 32 & $20 \mathrm{~h} 55 \mathrm{~m} 30.00 \mathrm{~s},+43 \mathrm{~d} 47 \mathrm{~m} 00.0 \mathrm{~s}$ & 16792320 \\
\hline P20015: 33 & $20 \mathrm{~h} 53 \mathrm{~m} 06.00 \mathrm{~s},+43 \mathrm{~d} 47 \mathrm{~m} 00.0 \mathrm{~s}$ & 16792576 \\
\hline P20015 : 34 & $20 \mathrm{~h} 50 \mathrm{~m} 42.00 \mathrm{~s},+43 \mathrm{~d} 47 \mathrm{~m} 00.0 \mathrm{~s}$ & 16792832 \\
\hline P20015 : 42 & $20 \mathrm{~h} 55 \mathrm{~m} 30.00 \mathrm{~s},+43 \mathrm{~d} 11 \mathrm{~m} 00.0 \mathrm{~s}$ & 16793088 \\
\hline P20015 : 43 & $20 \mathrm{~h} 53 \mathrm{~m} 06.00 \mathrm{~s},+43 \mathrm{~d} 11 \mathrm{~m} 00.0 \mathrm{~s}$ & 16793344 \\
\hline P00462 : 0 & $20 \mathrm{~h} 50 \mathrm{~m} 38.00 \mathrm{~s},+45 \mathrm{~d} 05 \mathrm{~m} 10.0 \mathrm{~s}$ & 24251392 \\
\hline P00462 : 1 & $21 \mathrm{~h} 01 \mathrm{~m} 13.00 \mathrm{~s},+44 \mathrm{~d} 54 \mathrm{~m} 43.0 \mathrm{~s}$ & 24251648 \\
\hline P00462 : 2 & $20 \mathrm{~h} 58 \mathrm{~m} 51.00 \mathrm{~s},+45 \mathrm{~d} 12 \mathrm{~m} 42.0 \mathrm{~s}$ & 24251904 \\
\hline P00462: 3 & $20 \mathrm{~h} 59 \mathrm{~m} 05.00 \mathrm{~s},+42 \mathrm{~d} 59 \mathrm{~m} 38.0 \mathrm{~s}$ & 24252160 \\
\hline P00462 : 4 & $21 \mathrm{~h} 00 \mathrm{~m} 17.00 \mathrm{~s},+43 \mathrm{~d} 23 \mathrm{~m} 25.0 \mathrm{~s}$ & 24252416 \\
\hline P00462 : 5 & $21 \mathrm{~h} 01 \mathrm{~m} 50.00 \mathrm{~s},+44 \mathrm{~d} 10 \mathrm{~m} 34.0 \mathrm{~s}$ & 24252672 \\
\hline P00462 : 6 & $20 \mathrm{~h} 50 \mathrm{~m} 19.00 \mathrm{~s},+42 \mathrm{~d} 58 \mathrm{~m} 37.0 \mathrm{~s}$ & 24252928 \\
\hline P00462 : 7 & $20 \mathrm{~h} 48 \mathrm{~m} 44.00 \mathrm{~s},+43 \mathrm{~d} 17 \mathrm{~m} 51.0 \mathrm{~s}$ & 24253184 \\
\hline
\end{tabular}

very similar to those for other star-forming regions observed in a similar manner-see, in particular, Figure 8 of Winston et al. (2007) for $3.6 \mu \mathrm{m}$; the NaNeb histograms indicate that our $90 \%$ completeness limits are about [3.6] $=15$ decreasing to about $[8.0]=12.5$. For our purposes, however, the more important number is the completeness for detecting objects in all four channels. We estimate this by determining where the four-channel differential source counts drop below the $3.6 \mu \mathrm{m}$ source counts by more than $20 \%$, which occurs at about [3.6] $=$ 12.2. This is about as expected given that four-channel catalog is normally limited by the detections in the $8.0 \mu \mathrm{m}$ channel. This completeness limit corresponds to a spectral type of M4 or M1 according to the BCAH98 isochrones (Baraffe et al. 1998) for 1 or $5 \mathrm{Myr}$, respectively, a distance of $600 \mathrm{pc}$ (assuming no reddening), and the temperature to spectral type scale defined by Luhman et al. (2003). The faintest object in our final catalog has [3.6] $\sim 16$, corresponding to a mass of $0.02-0.03 M_{\odot}$ at $1-5$ Myr age, assuming the $3.6 \mu \mathrm{m}$ flux is photospheric.

The remaining IRAC observations from program 462 were designed using the fixed-cluster observing mode to cover the irregularly shaped regions to the same depth as the main map and to create a final map that is approximately square in shape. The map center given in Table 1 is the approximate center of each AOR. We used the software developed by R. Gutermuth \& T. Megeath (2007, private communication) for use by the IRAC GTO and Gould's Belt teams to construct these AORs.

\subsection{Basic Data Reduction}

We started with the Spitzer Science Center (SSC) pipelineproduced basic calibrated data (BCDs), version S14.4. We ran the IRAC Artifact Mitigation code written by S. Carey and available on the SSC Web site. We constructed mosaics from the corrected BCDs using the SSC mosaicking and point-source extraction software package MOPEX (Makovoz \& Marleau 2005). The mosaics have a pixel scale of $1^{\prime \prime} .22$ pixel $^{-1}$, very close to the native pixel scale. Figure 2 shows the mosaics in channel $1(3.6 \mu \mathrm{m})$, and Figure 3 shows a three-color IRAC mosaic $(4.5,5.8$, and $8 \mu \mathrm{m})$.

We performed aperture photometry on the combined longand short-exposure mosaics separately using the output of 


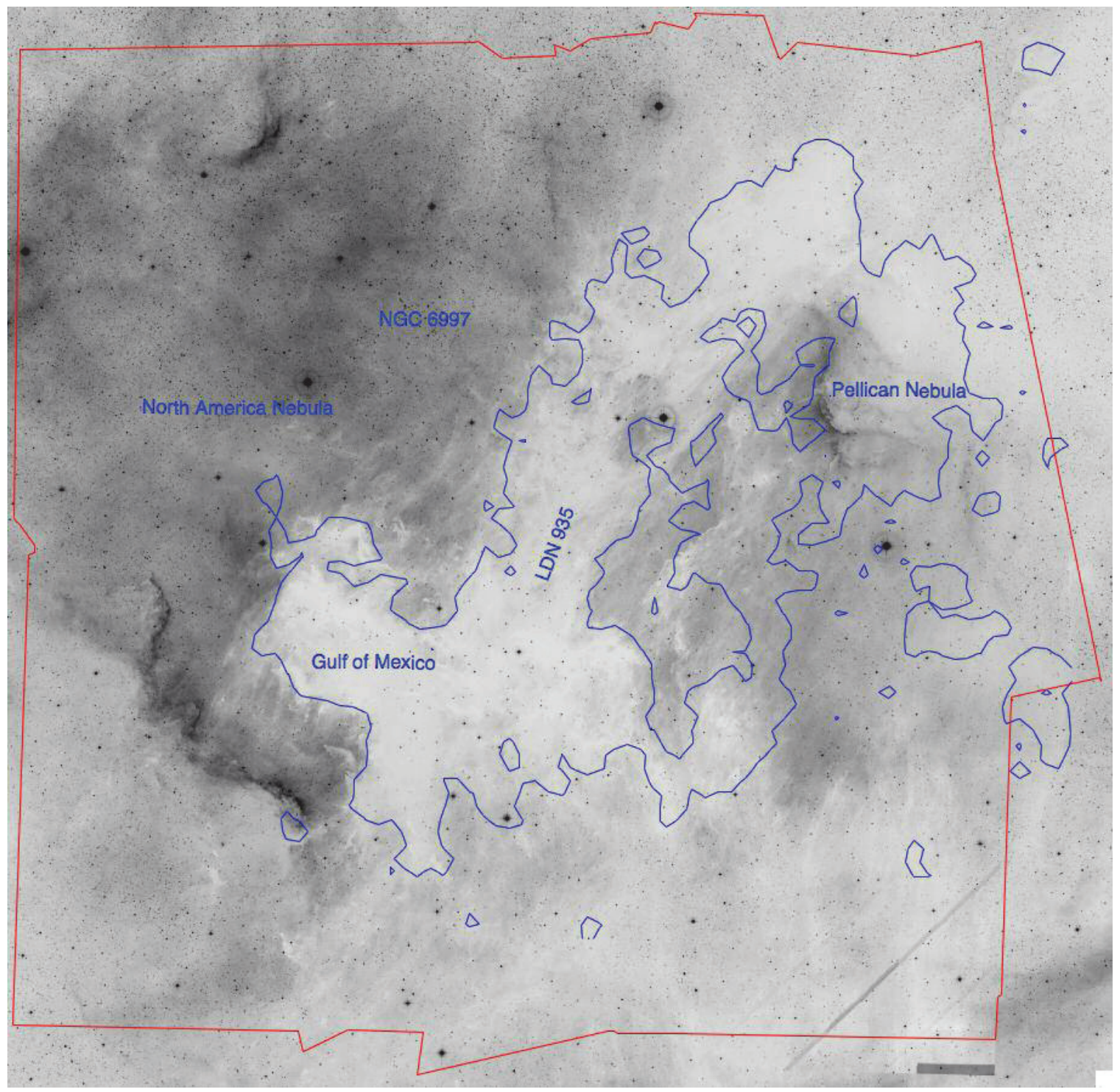

Figure 1. Optical image $(\sim 4 \times 3.2)$ of the NANeb region from the Palomar All Sky Survey. North is up, east is to the left. The left and right edges are at $21 \mathrm{~h} 02 \mathrm{~m} 32 \mathrm{~s}$ and $20 \mathrm{~h} 46 \mathrm{~m} 52 \mathrm{~s}$; the top and bottom edges are at $45 \mathrm{~d} 25 \mathrm{~m} 40 \mathrm{~s}$ and $42 \mathrm{~d} 40 \mathrm{~m} 56 \mathrm{~s}$. The region observed by IRAC is indicated by red lines. The green line is a smoothed $A_{v}=5$ contour from the Cambrésy et al. (2002) extinction map. The galactic coordinate of the center of this image is $l=84.8$ and $b=-0.6$.

(A color version of this figure is available in the online journal.)

the APEX (par of the MOPEX software) detection algorithm (APEX is a part of the MOPEX package) and the "aper" IDL procedure from the IDLASTRO library. We used a 2 pixel radius aperture and a sky annulus of 2-6 pixels. The (multiplicative) aperture corrections we used follow the values given in the IRAC Data Handbook: 1.213, 1.234, 1.379, and 1.584 for IRAC channels 1, 2, 3, and 4, respectively. Fluxes have been converted to magnitude given the zero magnitude fluxes of $280.9 \pm$ $4.1,179.7 \pm 2.6,115.0 \pm 1.7$, and $64.1 \pm 0.9$ for channels 1, 2, 3, and 4 respectively (IRAC Data Handbook). We take our photometry errors to be that given by the IDL procedure. We have checked that the uncertainties derived by the IDL procedure are consistent with the dispersion in IRAC colors for bright, off-cloud sources. For the longer integration times, the estimated average errors on our photometry in the four IRAC channels are $0.020,0.025,0.031$, and 0.035 mag for bright stars, increasing to $\sim 0.1 \mathrm{mag}$ for channels $1-4$ at $15,14.7,13.2$, and $12.7 \mathrm{mag}$.
Since the sources have been extracted individually from the mosaiced BCDs, we have used the overlap between BCDs to estimate our astrometric precision. The histogram of coordinate differences shows a $1 \sigma$ rms uncertainty of $0^{\prime} \cdot 3(\sim 1 / 4$ of pixel) in both directions.

The APEX source detection algorithm has a tendency to identify multiple sources within the PSF of a single bright source which can cause significant confusion at the bandmerging stage. Because our nominal fluxes are from the 2 pixel aperture photometry, any object which has a companion within 2 pixels will have a confused flux. The photometry lists were therefore cleaned of multiple sources prior to the band merging. Objects with a companion within 2 pixels had one of the sources removed. The source chosen for removal was the one with the lowest signal-to-noise ratio.

We extracted photometry from the long and short exposure mosaics separately for each channel, and merged these source lists together by position using a search radius of 2 pixels (2".44) 


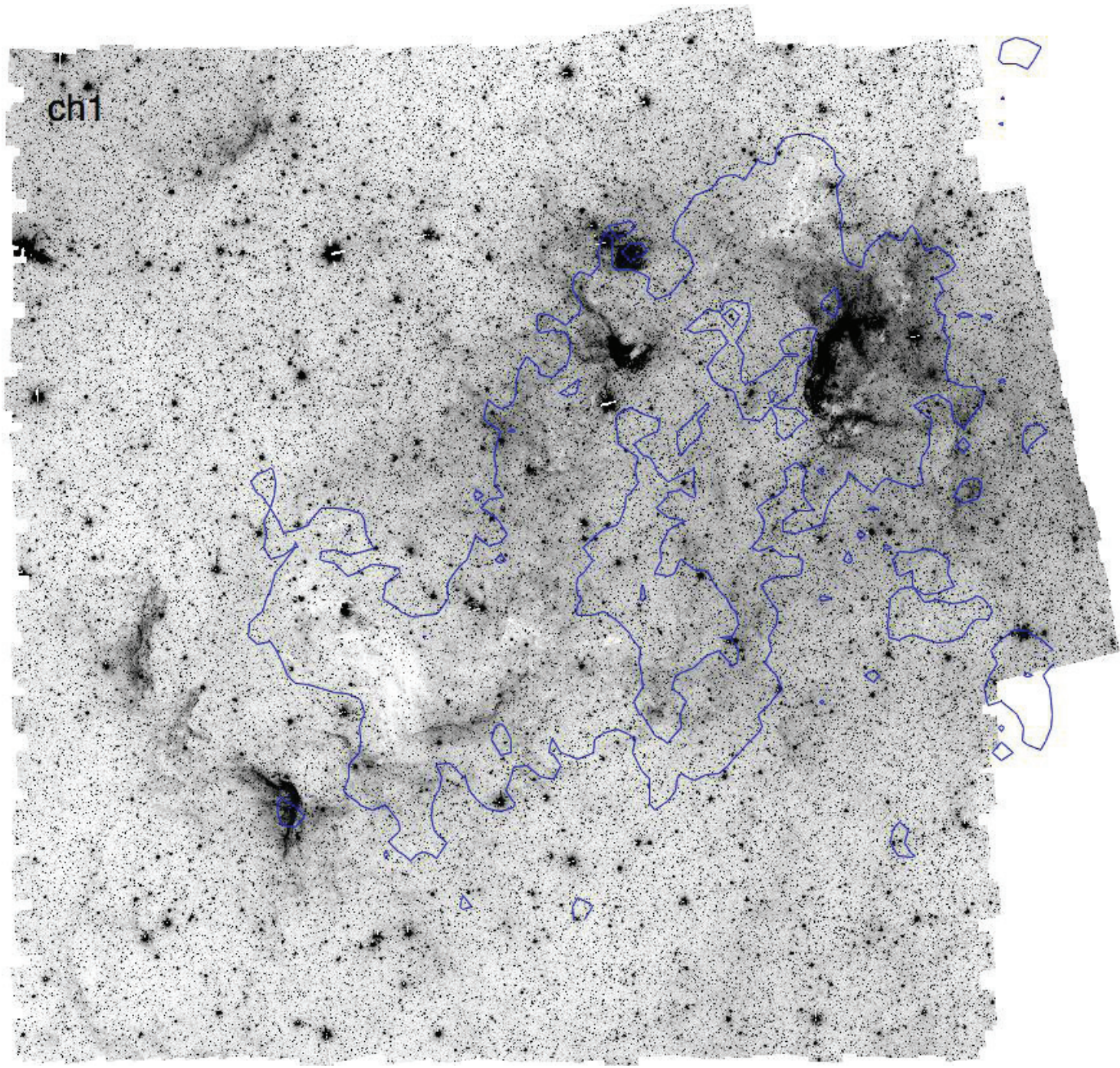

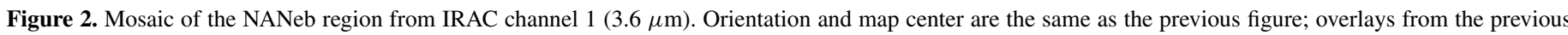
figure are included for reference.

(A color version of this figure is available in the online journal.)

to obtain a catalog for each channel. The magnitude cutoff where we transition to using the long rather than the short exposure photometry corresponds to magnitudes of $11,10,8.4$, and 7.5 mag for channels $1,2,3$, and 4, respectively.

Because of the complex nebulosity in this region, APEX (like any point-source detection algorithm) can be fooled by structure in the nebulosity. This effect is most apparent at $8 \mu \mathrm{m}$, where there are also the fewest stellar (point) sources detected. To attempt to remove false sources, we have compared photometry obtained via a 2 and 3 pixel aperture. After applying the aperture correction given in the IRAC Data Handbook, we eliminated sources with a difference of magnitude $>0.2 \mathrm{mag}$; doing so rejected $32 \%$ of the raw detections in IRAC's $8 \mu \mathrm{m}$ band. This process is only applied to the $8 \mu \mathrm{m}$ channel because the nebulosity is most prominent there (due to the presence of strong polycyclic aromatic hydrocarbon (PAH) bands at 7.6 and $8.6 \mu \mathrm{m})$. Also, because there are the fewest point sources detected in this band (see Table 2), the chances of there being two legitimate sources within 2-3 pixels of each other are much lower than in channels 1 or 2 . Spot-checking the images confirms this assertion. We acknowledge this step may eliminate some true YSOs from our four-band catalog. We deem this to be acceptable because our goal is to produce a minimally contaminated catalog of YSOs, not a complete catalog.

\subsection{Ancillary Data and Bandmerging}

Table 2 provides statistics for our entire catalog of objects; an object is included in our master catalog even if it is only detected in a single band. In order to create our final multiwavelength catalog which we will use to identify new YSOs, we first merged the four individual IRAC source lists together, starting with IRAC-1, taking the closest source within $1^{\prime \prime}$ as the best match. The radius of $1^{\prime \prime}$ has been chosen based on our astrometric precision of 0.3 (see section above) and the density of sources in the $3.6 \mu \mathrm{m}$ image (i.e., the most crowded image).

In order to provide photometry at other bands, we crossmatched our catalog to 2MASS, taking the closest source within $1^{\prime \prime}$, and then to our optical catalog, again taking the closest source within $1^{\prime \prime}$. Out of the 63084 objects detected at all four IRAC channels, $93 \%$ have a 2 MASS counterpart. For the entire region, $10 \%$ of the final catalog stars have an optical counterpart; out of just the region covered by the optical photometry data, $28 \%$ have an optical counterpart. The MIPS catalog will be discussed in 


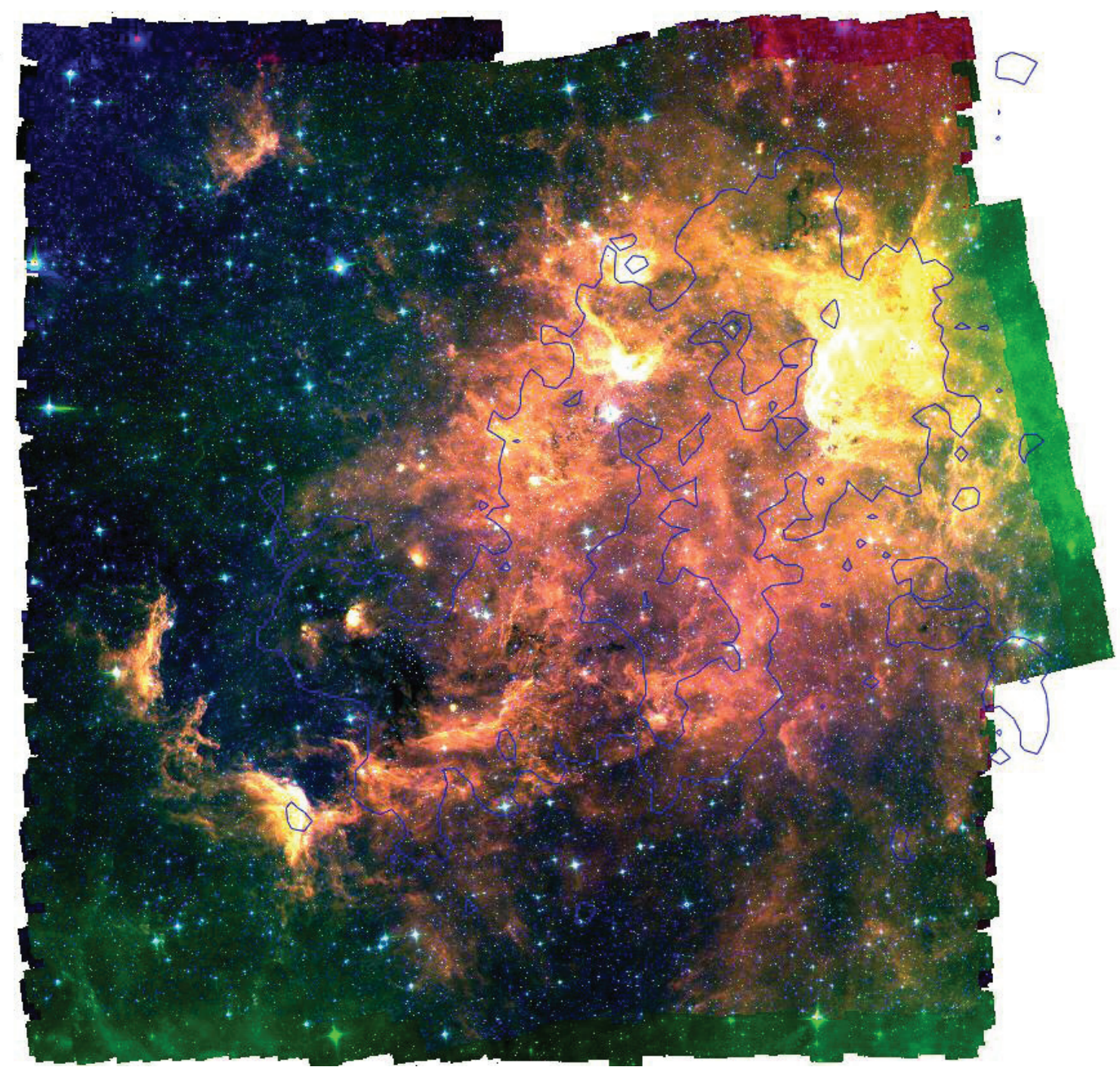

Figure 3. Three-color view of the IRAC NANeb with $4.5 \mu \mathrm{m}$ in blue, $5.8 \mu \mathrm{m}$ in green, and $8 \mu \mathrm{m}$ in red. Orientation and map center are the same as Figure 1; overlays from Figure 1 are included for reference.

Table 2

Number of IRAC Sources Extracted from the NANeb Data

\begin{tabular}{lcccccccc}
\hline \hline Band & Number of Sources & \multicolumn{6}{c}{ Fractional Number of Sources that Match (in \%) } \\
\cline { 3 - 8 } & & {$[3.6]$} & {$[4.5]$} & {$[5.8]$} & {$[8.0]$} & {$[3.6+4.5]$} & {$[3.6+4.5+5.8]$} & {$[3.6+4.5+5.8+8.0]$} \\
\hline$[3.6]$ & 558053 & $\ldots$ & 75 & 32 & 12 & 75 & 30 & 11 \\
{$[4.5]$} & 510922 & 82 & $\ldots$ & 33 & 13 & 82 & 33 & 12 \\
{$[5.8]$} & 200875 & 90 & 83 & $\ldots$ & 32 & 83 & 83 & 31 \\
{$[8.0]$} & 77898 & 84 & 83 & 82 & $\ldots$ & 83 & 61 & 81 \\
$J H K_{s}$ & 220799 & 90 & 86 & 65 & 27 & 86 & 71 & 39 \\
$B V I$ & 16595 & 85 & 83 & 72 & 40 & 83 & 48 & 43 \\
{$[24]$} & 4232 & 51 & 51 & 48 & 43 & 51 & 64 \\
\hline
\end{tabular}

Note. As an example of how to interpret this table, the "75" in cell ([3.6], [4.5]) means that $75 \%$ of the stars detected at $3.6 \mu \mathrm{m}$ also are detected at $4.5 \mu \mathrm{m}$.

detail in R09. In summary, we have covered approximately the same area as the IRAC map, and performed the source extraction using APEX.

$B V I$ images were obtained with the KPNO $0.9 \mathrm{~m}$ telescope on four photometric nights in 1997 June. An area of approximately $2.4 \times 1.7 \mathrm{deg}^{2}$ was covered by mosaicing the $23.2 \times 23.2 \mathrm{FOV}$
CCD in a grid with overlap of typically $3^{\prime}$ along each border. Exposures of 10 and $500 \mathrm{~s}$ enabled unsaturated photometry between $V=11$ and $20 \mathrm{mag}$. In IRAF, images were bias subtracted and flat fielded with sky flats taken each night. Sources were identified using a $5 \sigma$ detection threshold and the DAOFIND task. The photometry was measured through an 
aperture of 5 pixel radius with background determined as the median in an annulus from 7 to 14 pixels where the plate scale was 0.68 pixel $^{-1}$. Aperture corrections were applied. Absolute photometric calibration was achieved through observation of Landolt standards and further self-calibrated across the overlap regions using stars in common between frames. First, the short frames were calibrated to the long frames then the long frames were tied across the distinct telescope pointings, ignoring individual photometric errors larger than $0.1 \mathrm{mag}$. The average of the 43 spatial frame-to-frame offsets was 0.024 mag with dispersion $0.041 \mathrm{mag}$ and a few frames requiring offsets as large as $0.1 \mathrm{mag}$. The individual offsets themselves have standard deviation typically $0.02-0.03 \mathrm{mag}$ and standard deviation in the mean $<0.01 \mathrm{mag}$, which we take as the self-calibration error. Photometry was adopted from the long frames except when in the nonlinear or saturated regimes of the CCD response in which case the short frame photometry was adopted; these criteria were applied at $B<15.5, V<14.5$, and $I<13.5$. Astrometry was obtained using the Hubble Space Telescope Guide Star Catalog and the TASTROM task and is estimated accurate to $<0.3(1 \sigma)$ based on stars in the overlap regions.

Additional $R I$ frames were obtained on a fifth night over a more limited area, $2 \times 1.5 \mathrm{deg}^{2}$. These were reduced in a similar way, requiring a $5 \sigma$ detection threshold.

\section{IDENTIFICATION AND CHARACTERIZATION OF OUR YSO SAMPLE}

\subsection{Selection of YSO Candidates}

Several studies in the literature, usually focusing on subregions of this complex, have identified a total of $\sim 170$ YSOs in the area covered by our IRAC map, using a variety of techniques such as NIR excess or $\mathrm{H} \alpha$ emission. Most of these objects are earlier than K7. A complete list of the new Spitzer data for these previously known YSOs will appear in R09.

Now, with our new, comprehensive multiwavelength view of the complex, we can begin to create a census of the members of this complex having IR excesses. However, doing so is difficult, as it requires an extensive weeding-out of the galactic and extragalactic contaminants. To begin this task, we have opted to create a minimally contaminated sample of Spitzer-selected YSO candidates, as opposed to identifying every possible YSO candidate. By minimally contaminated sample, we simply mean a sample which includes as few non-YSOs as possible (i.e., from which asymptotic giant branch stars, active galactic nucleus (AGN), and any other objects whose IR colors mimic YSOs have been eliminated). We identify only stars with excesses at IRAC wavelengths as YSOs-hence, any member without excess (i.e., Class III YSOs) or stars with excess only at wavelengths greater than $8 \mu \mathrm{m}$ (inner cleared regions or transitional disks) will not be included. We now discuss the selection criteria we have used to select our reliable YSO sample.

Our selection method is described below and illustrated in Figure 4. We initially require detection at all four IRAC bands, which substantially limits the catalog (see Table 2) and then we follow the IRAC four-band source characterization described in Gutermuth et al. (2008, Section 4.1). Using their extragalactic contamination criteria, we have first rejected 272 sources as having colors consistent with galaxies dominated by $\mathrm{PAH}$ emission. This selection has been made in the [4.5]-[5.8]/[5.8][8] and [3.6]-[5.8]/[4.5]-[5.8] planes, where we reject objects seen in the gray zones plotted in panels (a) and (b) of Figure 4; this selection is defined by two sets of inclusive equations:

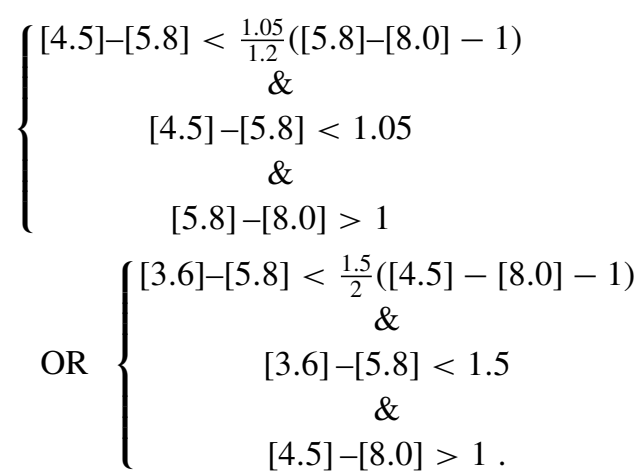

We then rejected 823 more sources having colors consistent with AGN in the [4.5] versus [4.5]-[8.0] plane; the region of color space used to select AGN-like sources is plotted in panel (c) of Figure 4 and defined by the following equations:

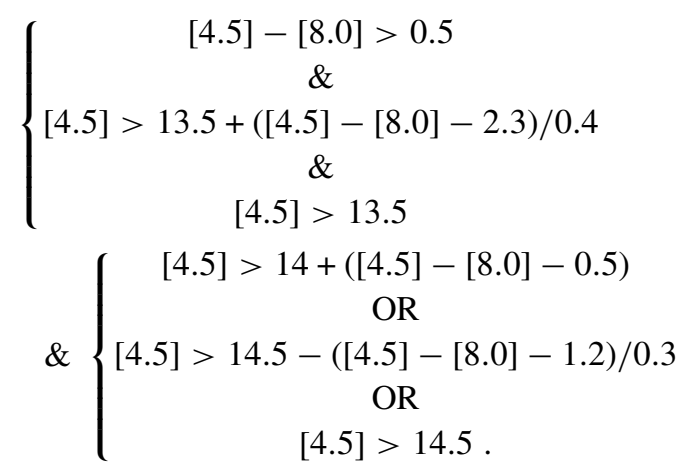

(see Appendix A of Gutermuth et al. 2008 for more details). Note that the AGN-like source selection has been made in the observed [4.5] versus [4.5]-[8.0] plane, whereas Gutermuth et al. (2008) use the dereddened plane. Gutermuth et al. (2008) are able to work easily in the dereddened plane because they have a high spatial resolution $A_{v}$ map. Because the NAN complex is much further away than NGC 1333, we do not have the ability to construct such a high-resolution $A_{V}$ map. We tested the implications of this limitation by using the Cambrésy et al. (2002) extinction map to deredden individual objects. Just 25 sources would be dropped by performing this selection in the dereddened plane; they are fairly uniformly spread over the entire mapped region (and hence are likely contaminants rather than YSOs). Just three of these are in the Gulf of Mexico region (see R09) where we do not expect very much background contamination due to the very high reddening. Satisfied that this decision does not significantly affect our final sample of YSOs, we have chosen to keep our source selection in the observed plane.

Once these background contaminants are removed from consideration, we are left with a population of 61,989 IRAC sources, dominated by objects with the apparent colors of stellar photospheres. As in Gutermuth et al. (2008), we have further selected YSO candidates in the [4.5]-[8] versus [3.6]-[5.8] color-color diagram meeting the following criteria:

$$
\left\{\begin{array}{c}
{[4.5]-[8]>0.5} \\
\& \\
{[3.6]-[5.8]>0.35} \\
\& \\
{[3.6]-[5.8] \leqslant \frac{0.14}{0.04} \times([4.5]-[8]-0.5)+0.5 .}
\end{array}\right.
$$



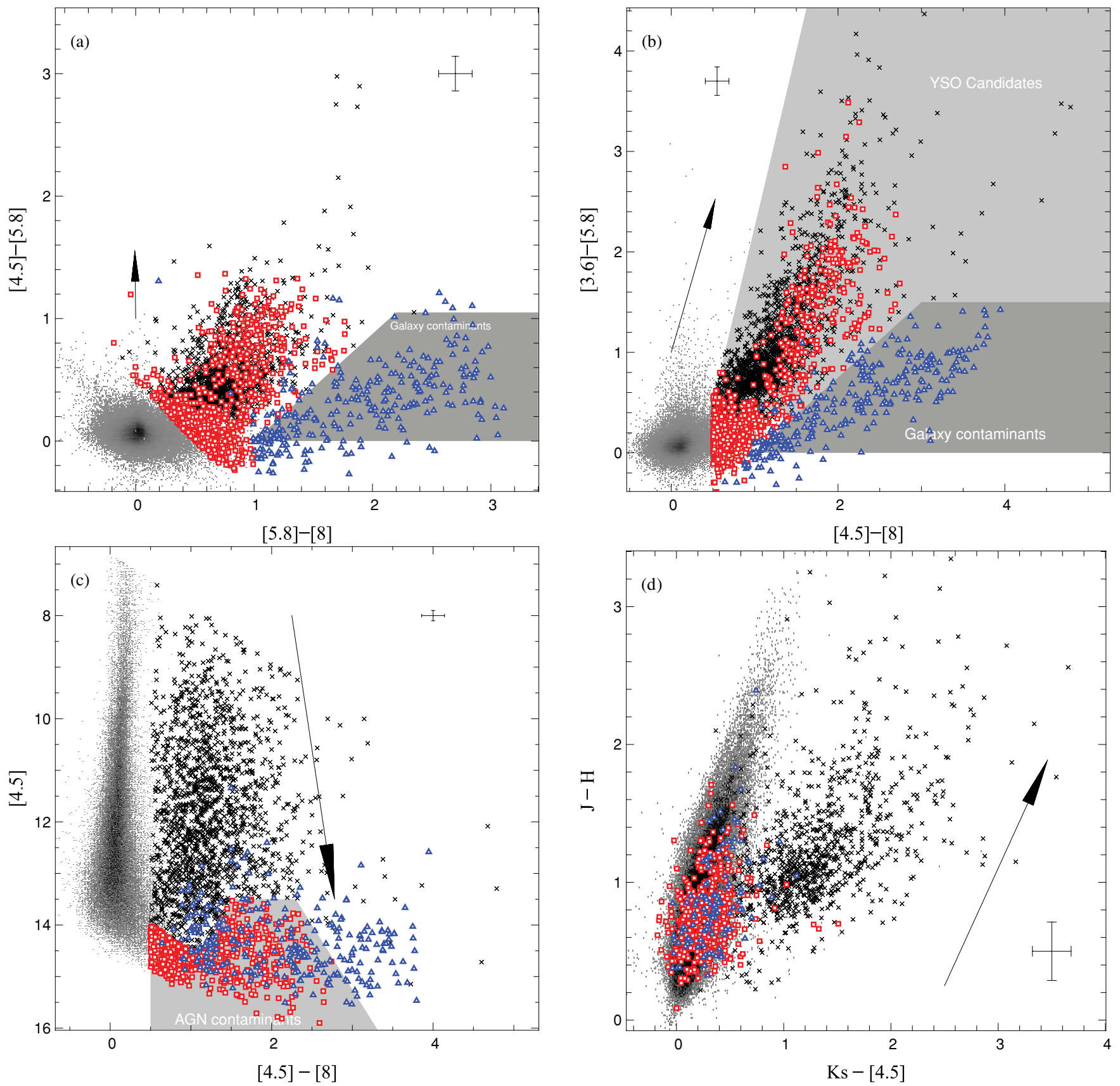

Figure 4. IRAC color-color and CMDs used to reject background contaminants panels $(a)-(c)$ and select YSOs candidates panel $(b)$. An additional diagram shows the distribution of background contaminants and YSO candidates in a 2MASS-IRAC color-color diagram limited to targets with 2MASS counterparts panel $(d)$. In all diagrams, blue triangles are sources flagged as PAH-emission sources (galaxies) located in the darkest gray area of panel $(a)$ or $(b)$. Red squares are sources flagged as AGN, selected in the gray area of panel $(c)$. Finally, YSO candidates are plotted with black cross symbols; they have been selected in the brightest gray area of panel $(b)$ if not previously flagged as background contaminants. The reddening vectors correspond to an $A_{K}$ of 10 in panels $(a),(b)$, and $(c)$, and $A_{K}$ of 2 in panel $(d)$. We averaged the extinction law given in Flaherty et al. (2007) for Serpens, Orion, and Ophiuchus.

(A color version of this figure is available in the online journal.)

This selection is indicated in Figure 4, panel (b). This leaves 1657 candidates.

Continuing to follow Gutermuth et al. (2008), we used their selection criteria to identify objects from the 1657 candidates whose IRAC fluxes may be contaminated by emission lines from shocks given their [3.6]-[4.5] and [4.5]-[5.8] colors by the equations

$$
\left\{\begin{array}{c}
{[3.6]-[4.5]>\frac{1.2}{0.55}([4.5]-[5.8]-0.3)+0.8} \\
\& \\
{[4.5]-[5.8] \leqslant 0.85} \\
\& \\
{[3.6]-[4.5]>1.05}
\end{array}\right.
$$


We found only two YSO candidates that matched these criteria (205608.3+433654.2 and 205702.1+433431.3). Their spectral energy distributions (SEDs) are compatible with their being real YSOs whose aperture photometry is affected by emission from a compact, circumstellar nebula, causing an excess at $4.5 \mu \mathrm{m}$ relative to the two adjoining IRAC bands. Moreover, these two objects are located inside the "Gulf of Mexico" where the concentration of YSOs is the highest in the cloud, and where previous optical emission-line surveys have found numerous $\mathrm{HH}$ regions. Given these considerations, we retain these objects in our YSO candidate list.

Of the 1657 YSO candidates, $972(59 \%)$ have a 2MASS counterpart. Out of the entire region, 131 (7.9\%) have an optical counterpart; out of the region covered by the optical data, $10.4 \%$ have an optical counterpart. All but 63 of the YSO candidates are inside the region covered by MIPS data, and $45 \%$ of the candidates have a MIPS $24 \mu \mathrm{m}$ counterpart. We note that the percentage of 2MASS and optical YSO counterparts is less than for the whole IRAC four-band catalog $(92 \%$ and $28 \%$, respectively, see Section 2.3); this is due to the fact that YSOs are mostly located inside highly reddened regions and not detected in our shorter wavelengths. With this selection of $\sim 1600$ YSOs, we have increased the number of likely YSOs in the NANeb region by an order of magnitude.

We compare the positions of both the YSO candidates and background contaminants in a 2MASS/IRAC color-color diagram in panel (d) of Figure 4. A large fraction $(90 \%)$ of sources flagged as galaxies or AGN fall in the region occupied by reddened main-sequence stars, and hence blueward in $K_{s}-$ [4.5] color from the YSOs. Just 5\% of the YSO candidates are located in this same region, two-thirds of which are classified as intermediate between Class II and Class III (see Section 3.3).

It is hard to estimate the contamination fraction of our YSO candidates without additional data (e.g., spectroscopic or Xray confirmation). However, we can make a few worst case estimates. Because our survey area is fairly large, it includes some sections which are outside the main star-forming region and have relatively few candidate YSOs. The northeast corner of our survey region (see Figure 3), for example, offers one such relatively YSO-poor area. Specifically, we define a "field" region as being the area above a line from the midpoint of the east edge of Figure 3 to the midpoint of the north edge of the figure. If all the candidate YSOs in this region are contaminants, we derive a contaminant surface density of 32 objects per square degree. Assuming the AGN and other contaminants are uniformly distributed over our survey region, this yields an upper limit to the fraction of contaminants in our survey of $17 \%$.

\subsection{NANeb Age Estimation}

We have used optical photometry in order to constrain the age of the NANeb complex. Figure 5 shows the location of YSOs with optical and 2MASS photometry in an optical color-magnitude diagram. Also shown are Siess et al. (2000) isochrones, where the color- $T_{\text {eff }}$ conversion has been tuned so that the $100 \mathrm{Myr}$ isochrone follows the single-star sequence in the Pleiades (Stauffer 1996; Jeffries et al. 2007). The number of YSOs in this diagram is limited by the fact that it requires optical counterparts. This means that most of sources from this diagram are located inside the less-extincted regions of the NANeb, on the edge of the Pelican Nebulae $80 \%$ are located inside region of $A_{v}<5$, according to the Cambrésy et al. 2002 extinction map). The embedded Class I sources are not well detected in the optical data, and our YSO selection criteria do not allow

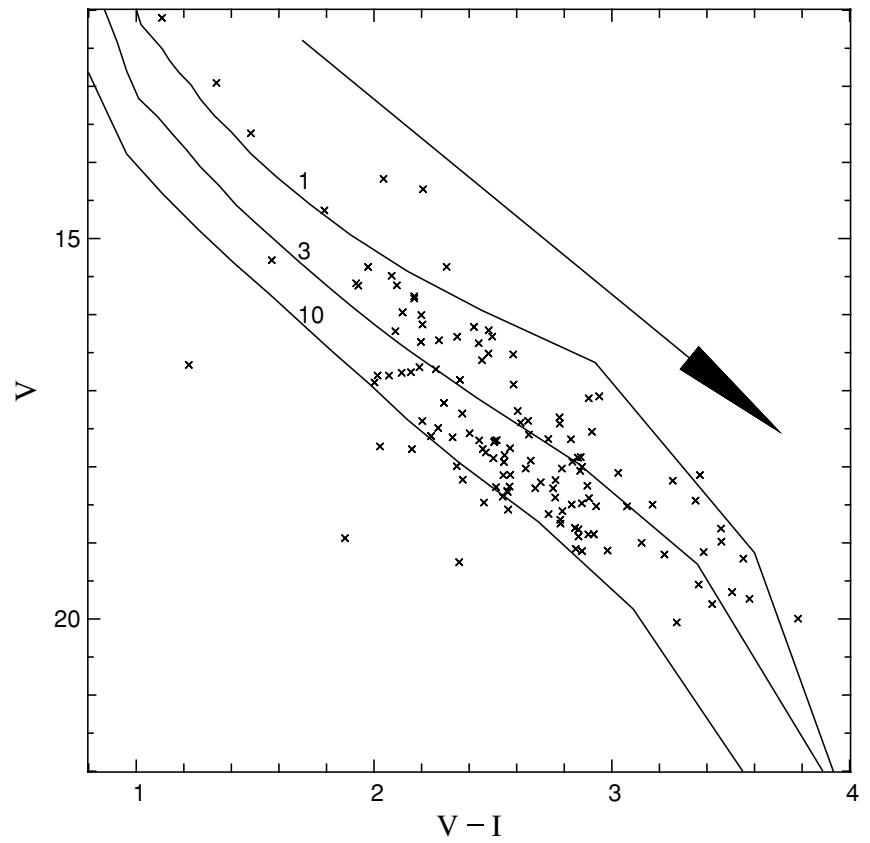

Figure 5. $V / V-I$ color-magnitude diagram for our YSO candidates. The solid lines are, from right to left, tuned Siess models (see the text) for 1, 3, and $10 \mathrm{Myr}$ scaled to the NANeb distance. A reddening vector of $A_{v}=5$ is plotted.

us to detect Class III sources, so this sample is mostly limited to Class II sources.

Our age estimates are uncertain for all of the normally expected reasons (e.g., uncertainties in the isochrones and their transfer to the observational plane; the need for and imprecision of the reddening corrections; the effect of spots and UV excesses on the estimated colors and effective temperatures of our target stars; binarity). However, it is apparent in Figure 5 that most of our YSO candidates (with optical counterparts) are younger than $10 \mathrm{Myr}$ according to the isochrone tracks. The median age is slightly older than $3 \mathrm{Myr}$, but the most embedded and probably youngest regions of NANeb are excluded from this optical color-magnitude diagram (CMD). The median age and the age dispersion is comparable to what found in NGC 2264 by Rebull et al. (2002). Since the reddening vector is essentially parallel to the isochrones, and YSOs with optical counterpart have low reddening, the median age will be minimally affected by reddening.

\subsection{Classes of YSO Candidates}

We classified this set of highly likely NANeb YSOs, according to the Lada (1987) Class I/II/III system, updated by André (1994), where the "Flat" class has been added between Class I and II. Classes are assigned based on the spectral index, defined by

$$
\alpha=\frac{d \log \lambda F(\lambda)}{d \log \lambda},
$$

where $F(\lambda)$ is the flux at the wavelength $\lambda$. For Class I, $0.3 \leqslant \alpha$; for Flat sources, $-0.3 \leqslant \alpha<0.3$; for Class II, $-1.6 \leqslant \alpha<-0.3$; and for Class III, $\alpha<-1.6$. Note that because we are making a Spitzer-based selection of YSO candidates, by definition none of our candidates will have a photospheric slope, so our Class III inventory is guaranteed to be incomplete. Further observations at other wavelengths (e.g., with Chandra) are required to find such objects. In the following, 


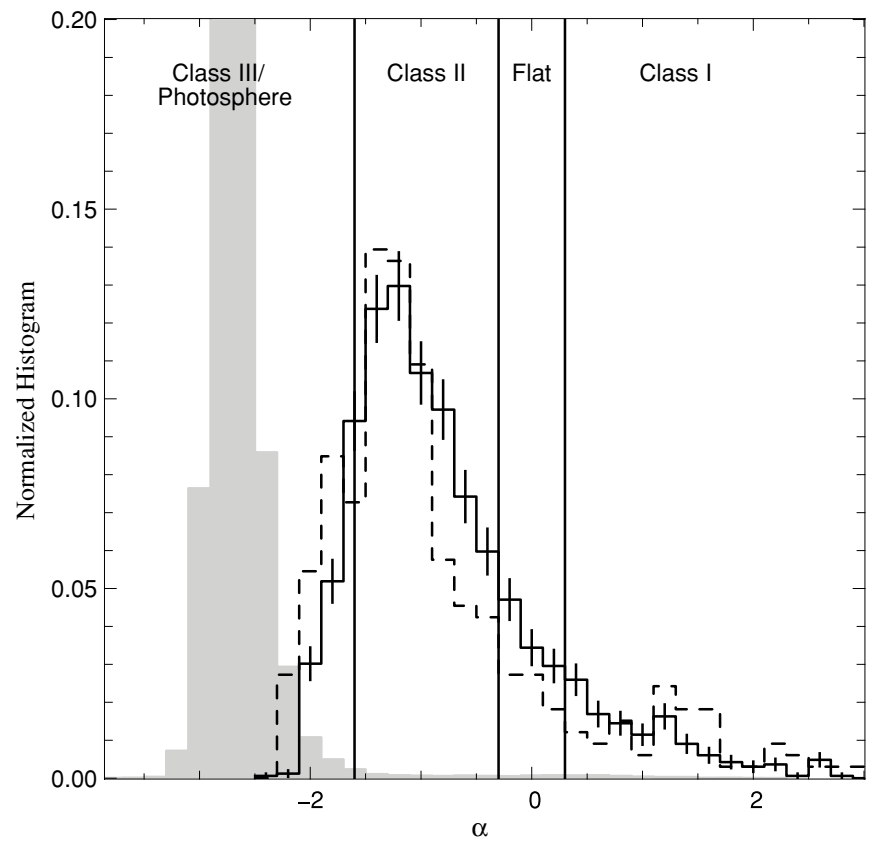

Figure 6. Normalized histograms of the distribution of spectral slope, $\alpha$. The continuous line is the histogram for the YSO candidates for the whole sample. All other IRAC sources from the NANeb are also indicated (in gray), as are the 330 YSO candidates we have found from the Harvey et al. (2006) highly reliable catalog (dashed line).

to avoid confusion, we call our YSO candidates with $\alpha<-1.6$ Class II-III.

We fit $\alpha$ over the four IRAC channel points from 3.6 to $8 \mu \mathrm{m}$. From the total 1657 YSO candidates, we found that 1059 (64\%) are Class II, $184(11 \%)$ are Flat, and $198(12 \%)$ are Class I. We classified the remaining $216(13 \%)$ YSOs as Class II-III because they have $\alpha$ between stellar photospheres $(\alpha=-2.7)$ and Class II $(\alpha=-1.6)$. A histogram of $\alpha$ for our YSO candidate sample is plotted in Figure 6. This figure also contains, for comparison, a histogram of the values of $\alpha$ for our entire NANeb catalog (mostly field stars), and that for the YSO candidates derived from the highly reliable Serpens catalog obtained by Harvey et al. (2006, 2007a, 2007b; see discussion below).

Plots similar to those appearing here (or in R09) can be found in most of the $\mathrm{c} 2 \mathrm{~d}$ series of papers on Serpens, Perseus, Ophiuchus, Chamaeleon II, and Lupus. We picked Serpens for our primary comparison here because it is thought to be similar in age to the NANeb, it contains a deeply embedded cluster similar to the Gulf of Mexico, it is in the Galactic plane $(l=32$, $b=+5)$, and Harvey et al. (2006, 2007a, 2007b) performed a Spitzer-specific source selection. However, there are some important differences. The Spitzer maps of Serpens are only $0.85 \mathrm{deg}^{2}$, nearly six times smaller than our map. The selection method for finding YSO candidates is much different in Harvey et al. (2006, 2007a, 2007b), for example, requiring a MIPS-24 detection, and the differences are most strongly apparent in the selection of Class III objects.

In order to fairly compare our data with the Serpens data, we used the highly reliable Serpens catalog (of all objects, not just their YSO candidates) provided by the $\mathrm{c} 2 \mathrm{~d}$ team as part of their final Legacy delivery (available on the SSC website) and applied our selection method to those data. From the sample of Serpens YSOs which pass our selection criteria, we calculated $\alpha$ in the same way that we did for the NANeb, from 3.6 to $8 \mu \mathrm{m}$. It is these values which appear in Figure 6. Our selection and classification scheme yield 52 Class I, 24 Flat disk, 189 Class II, and 65 Class II-III Serpens YSOs. The number of Class II-III objects is hard to compare to that found in Harvey et al. (2006) since they use the $24 \mu \mathrm{m}$ data in their classification scheme; but the number of Class I, Flat, and Class II are roughly comparable: 30, 33, and 163, respectively, in Harvey et al. (2006).

As a means to estimate a comparative evolutionary age for the NANeb stars, one can determine the ratio of the number of Class II ("older") to Class I or Flat sources ("younger"), and compare that ratio to that derived for other clusters, such as Serpens. For our entire NANeb YSO sample, we derive a ratio $N($ Class II $) / N($ Class I+Flat $)=1059 / 382=2.78 \pm 0.17$. Using our similarly analyzed data for Serpens, we derive this ratio as $2.49 \pm 0.33$ (roughly comparable to what was derived by Harvey et al. 2006; $2.6 \pm 0.4$, where they used 2MASS through $24 \mu \mathrm{m}$ data to compute alpha). The ratios for NANeb and Serpens are comparable, suggesting that the mean ages of the two YSO samples are indeed similar.

Harvey et al. (2006, 2007a, 2007b) used a series of Spitzerbased selection criteria to select a sample of YSOs; their final selection in Harvey et al. (2007a) primarily uses color-color and CMD to assign a likelihood that a given object is extragalactic. The process starts with requiring detection in all four IRAC bands as well as MIPS-24. Only about half of our minimally contaminated YSO sample has a MIPS-24 detection, so the Harvey et al. selection process by its very nature could only retrieve about half of our sample. However, $90 \%$ of our highly reliable sample with MIPS-24 detections is also retrieved by a Harvey et al. style source selection.

R09 contains a special discussion of the Gulf of Mexico, which is an interesting area containing hundreds of deeply embedded young stars, mostly associated with three subclusters. We note here that our requirement for having all four bands of IRAC for our source selection omits many of the objects in this region, and this requirement will be relaxed in R09 to find cluster members. However, using our selection criteria and our best possible YSO sample (as defined above) results in a ratio of $N($ Class II $) / N($ Class I+Flat) that is statistically significantly different inside the Gulf cluster than outside of it. The ratio, with its Poissonian error, is $901 / 261=3.45 \pm 0.24$ outside the Gulf of Mexico and 158/121 = $1.31 \pm 0.16$ inside this region. Moreover, we find comparatively very few Class II-III objects in the Gulf of Mexico region. Both these findings indicate that the Gulf of Mexico cluster is, in evolutionary terms, the youngest region within the NAN complex.

We explore now, how the NANeb reddening can change our YSO classification. Indeed, Muench et al. (2007), Appendix A, demonstrated that for a deeply embedded cluster $\left(A_{v} \sim 40\right)$, the IRAC SED slope of a typical K6 Class II member of IC 348 would be reddened into an apparent Flat spectrum. According to the Cambrésy et al. (2002) extinction map, however, 97\% of YSOs are located in region where the $A_{V}$ is less than 10 . Since the Cambrésy et al. (2002) map yields extinctions up to about 30 , we assume that for extinctions $\leqslant 10$ the predictions should be reliable (i.e., not "saturated" because the extinctions are so high that no background stars are present within the grid point). To test the effect of reddening on our classification, we have dereddened our SEDs prior to the spectral slope calculation, according to the Cambresy et al. map. The numbers of Class I, Flat disk, and Class II given by the dereddened SEDs-186, 177 , and 1043 - are quite similar to those using the observed SEDs-198, 184, and 1059, making the $N$ (Class II) $/ N($ Class I+Flat) ratio equal to $2.87 \pm 0.17$ instead of $2.78 \pm 0.17$. These 


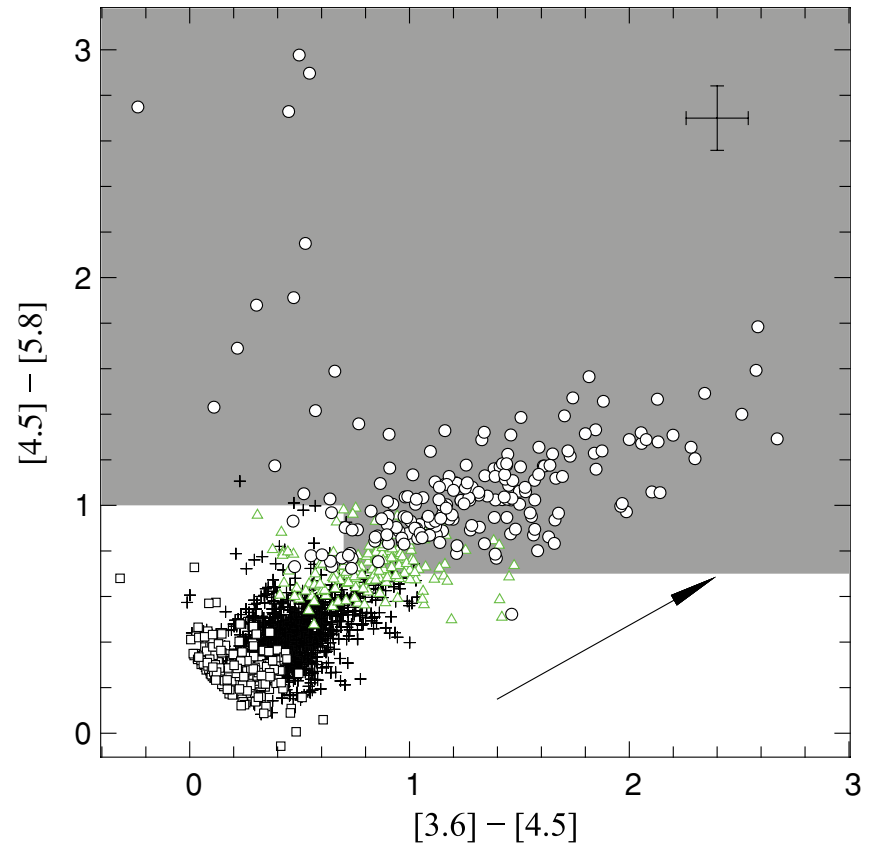

Figure 7. Spitzer color-color diagram. Open squares are Class II-III, plus symbols are Class II, green triangles are classified as Flat-spectrum sources, and circles are Class I. The green area is the protostar selection area defined by Gutermuth et al. (2008). A reddening vector of $A_{k}=10$ is plotted

(A color version of this figure is available in the online journal.)

small differences do not change our main conclusions about the spatial distribution of Class I and Class II.

There is another means by which we can gauge the effect of extinction on our YSO classification. Gutermuth et al. (2008) distinguished protostars and Class II by there [4.5]-[5.8] color excess. They chose this criterion specifically because it is relatively immune to extinction (because the interstellar extinction curve is relatively flat between these two wavelengths). Gutermuth et al. (2008) selected protostars as sources matching the following equation:

$$
\left\{\begin{array}{c}
{[4.5]-[5.8]>1} \\
\text { OR } \\
{[4.5]-[5.8]>0.7 \&[3.6]-[4.5]>0.7 .}
\end{array}\right.
$$

The Gutermuth et al. (2008) classification scheme does not include flat-spectrum sources as a separate class. Figure 7 shows the location of Class I/Flat/Class II and Class II-III YSOs in the [4.5]-[8.0]/[3.6]-[4.5] plane superimposed on the protostar area defined above. The location of our Class I and Class II sources agrees well with the regions defined in this diagram: 95\% of our Class I and 99\% of our Class II sources would be classified as protostars and Class II by the Gutermuth et al. criteria. Our Flat-spectrum sources lie on the border between Class I and Class II, with roughly equal numbers on either side of the Gutermuth et al. (2008) boundary.

\subsection{Spatial Distribution of YSO Candidates}

Now that we have a minimally contaminated sample of YSO candidates, we can look at their spatial distribution and investigate the degree of clustering and the relative spatial distribution of YSOs as a function of their class. We have computed a YSO density map of our observed region. We used a Kernel method (Silverman 1986) which yields a smooth isodensity contour map from the projected position of objects.
In each point of the space $(\alpha, \delta)$, the density is set, due to the contribution of all $n$ points, by the kernel density estimator:

$$
D(\alpha, \delta)=\frac{1}{h^{2}} \sum_{i=1}^{n} K\left(\alpha, \alpha_{i}, \delta, \delta_{i}\right)
$$

where $K$ is the kernel. For the kernel, we adopted a Gaussian shape:

$$
K\left(\alpha, \alpha_{i}, \delta, \delta_{i}\right)=\frac{1}{2 \pi} \exp \frac{-\left(\left(\delta-\delta_{i}\right)^{2}+\left(\alpha-\alpha_{i}\right)^{2} \cos ^{2} \delta\right)}{2 h^{2}},
$$

where $h$ is the smoothing parameter. We adopted $h=0.05$ $(0.5 \mathrm{pc})$, which is approximately the size of the smallest group of YSOs in the NANeb discernible 'by eye'.

Figure 8 shows contours of the density map we obtained, where both background contaminants and YSO candidates are plotted in two different panels. Using these density contours, we find first that the background contaminants (galaxies or AGN) are relatively evenly distributed across the FOV. However, we notice a lack of background contaminant sources on the central dark cloud. The lack of contaminants in the high-extinction regions can be quantitatively explained simply due to the fact that our survey is magnitude limited, and the extra extinction causes the observed fraction of galaxies to drop out of our sample. We checked this assumption by creating an artificial map of randomly distributed background sources reddened by the extinction map of Cambrésy et al. (2002). We then applied a magnitude cut to this artificial sample, corresponding to the effective faint limit imposed by our four-band YSO selection criteria. The resulting spatial distribution of selected galaxies mimics well the observed galaxy distribution in Figure 7 (top), confirming our assumption.

Our YSO candidates are located primarily on the central dark cloud (Lynds 935), and for the most part highly clustered. Further, we find that half of our YSO population is located in regions denser than $1000 \mathrm{YSOs} / \mathrm{deg}^{2}\left(\sim 10^{5} \mathrm{YSOs} / \mathrm{pc}^{2}\right)$ and cover a relatively small fraction of the sky $\left(\sim 0.5 \mathrm{deg}^{2}\right.$ compared to the $\sim 9 \operatorname{deg}^{2}$ observed).

From the distribution of YSOs, we distinguished by eye eight main clusters (for a discussion of more quantitative means to identify clusters of YSOs from similar catalogs, see, e.g., Jørgensen et al. 2008). We determined the center of each cluster as the local peak of the YSO density. We arbitrarily define the boundaries of these clusters as the YSO density contour at a level of one-fourth of the maximum density peak. The location, the size, the number of stars, and the mean $A_{v}$ for each cluster are provided in Table 3. Five of these clusters have been previously identified by Cambrésy et al. (2002); they are the most prominent clusters located inside the Gulf of Mexico and in the central part of the NANeb. Figure 9 shows the location of both our clusters and clusters defined by Cambrésy et al. One can see in this figure that cluster 4 of Cambrésy et al. (2002) does not exist in our YSO candidate distribution. Clusters 7 and 8 of Cambresy appear in our YSO distribution, but are similar to many other small clusters in the NANeb; they are not as densely populated as the ones we have defined. Because the Cambrésy et al. (2002) technique is sensitive to both WTTs and CTTs, whereas ours essentially excludes WTTs, it is reasonable that our two methods will yield somewhat different results. It is likely that the clusters found by Cambrésy et al. (2002) but not by us may be the evolutionarily older clusters in the region.

In the bottom panel of Figure 8, we plot the location of YSOs with different symbols corresponding to their class. To 

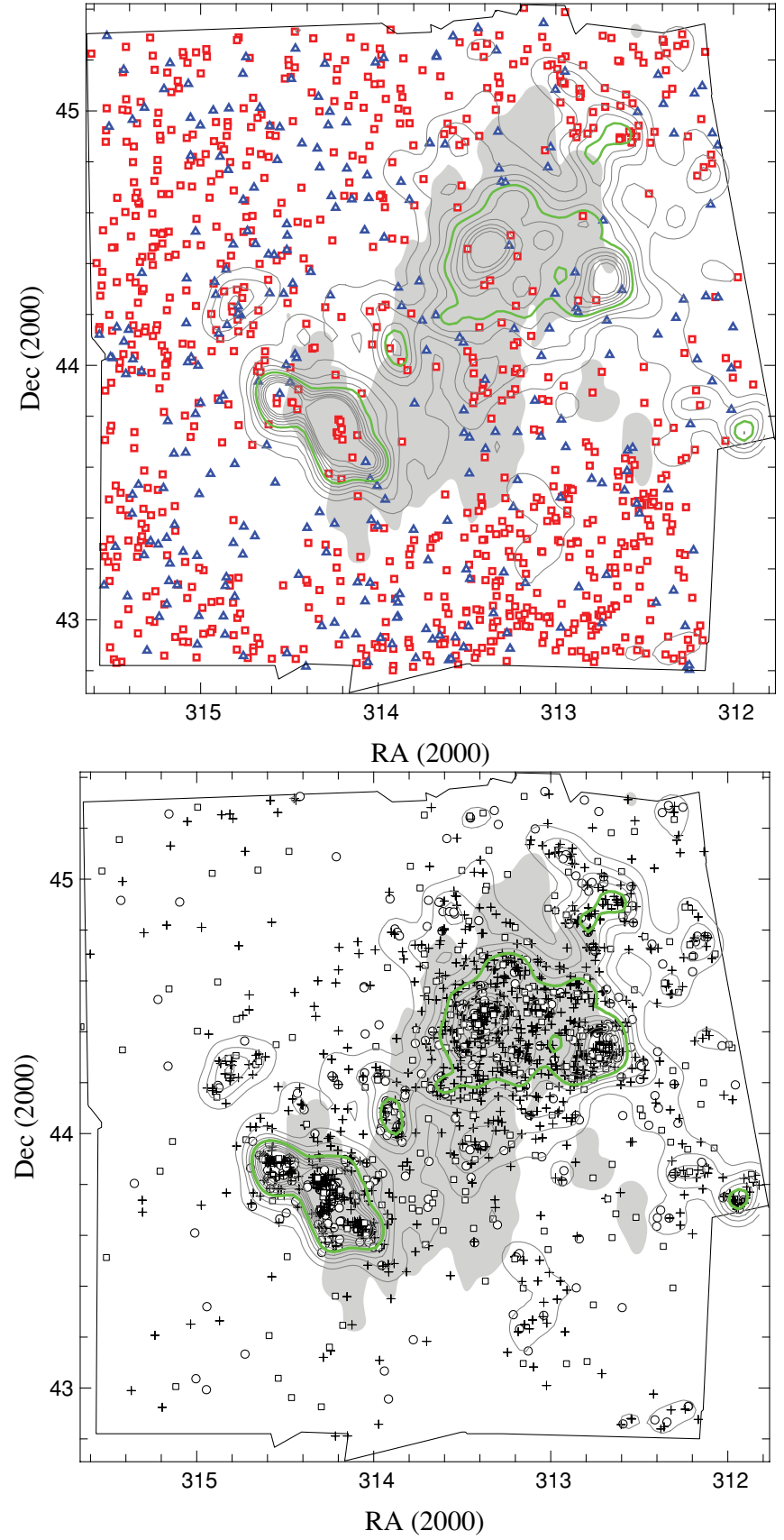

Figure 8. Top: the spatial distribution of background contaminants (red squares: AGN-like sources, blue triangles: galaxies; see the text and Figure 4). Bottom: the spatial distribution of YSO candidates, where symbols denote the object Class (circles: Class I or Flat, +: Class II, square: Class II-III). In both panels, we plot contours of YSO density (see the text for more details); the contour levels correspond to values plotted in Figure 10, from 200 to 2000 YSOs/deg 2 . The contour which surrounds half of the total YSO population $(\sim 1000$ YSOs $/ \mathrm{deg}^{2}$ ) is highlighted in green and with a thicker contour line. The background gray area indicates the $A_{V} \geqslant 5$ dark cloud from the Cambrésy et al. (2002) map.

(A color version of this figure is available in the online journal.)

investigate in more detail, a possible spatial segregation between Class I(+Flat), II, and II-III, we used the YSO density contours to define regions within which we have computed the ratio of $N($ Class I + Flat $) / N($ Class II) and $N($ Class II $) / N($ Class IIIII). The behavior of these ratios as a function of the density contour limit appears in Figure 10. This Figure shows that in dense regions (i.e., more clustered regions), the proportion of

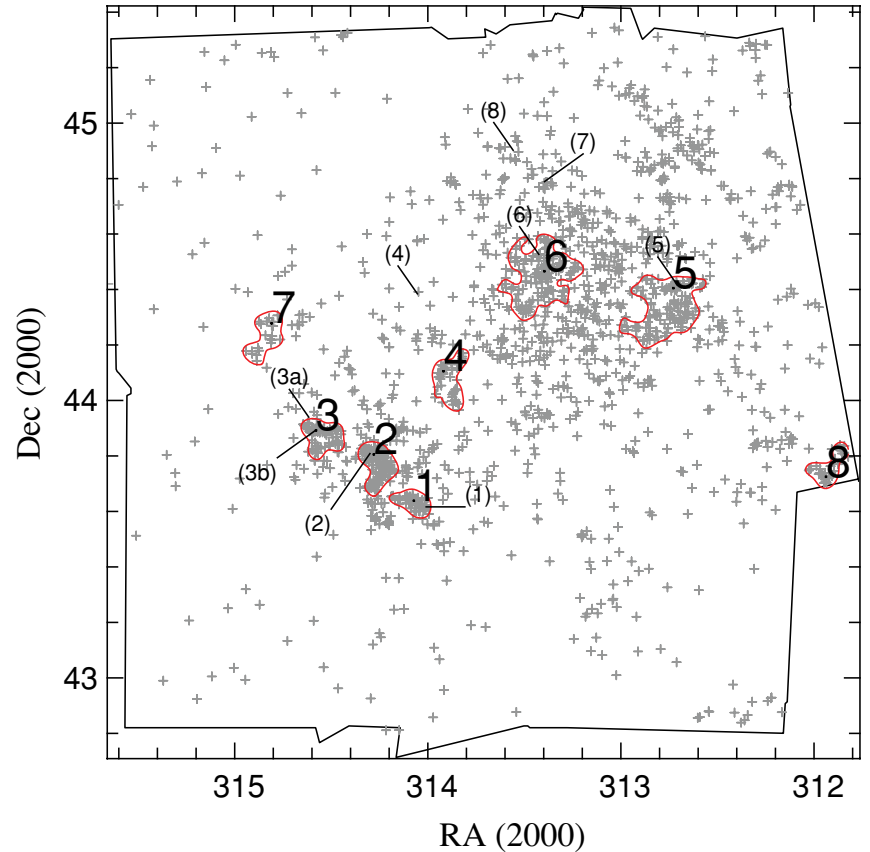

Figure 9. Location of clusters shown on the distribution of the $\sim 1600$ YSO candidates (plus symbols). The solid red lines are contours of clusters we adopted (see the text for more details). Our eight clusters are labeled with large, bold numbers, and no brackets. The location of clusters found by Cambrésy et al. (2002) is also indicated by smaller bracketed labels.

(A color version of this figure is available in the online journal.)

Table 3

Id, Center Coordinate, and Area of Identified Clusters are Indicated on the

First Four Columns, Followed by the Number of YSO Members and the

Average Reddening from the Extinction Map of Cambrésy et al. (2002)

\begin{tabular}{ccccccc}
\hline \hline Id & $\begin{array}{c}\text { R.A. } \\
\mathrm{J}(2000)\end{array}$ & $\begin{array}{c}\text { Decl. } \\
\mathrm{J}(2000)\end{array}$ & $\begin{array}{c}\text { Area } \\
\left(\mathrm{deg}^{2}\right)\end{array}$ & $\begin{array}{c}\text { YSOs } \\
(\text { count })\end{array}$ & $\begin{array}{c}\left\langle A_{v}\right\rangle \\
(\mathrm{mag})\end{array}$ & idc \\
\hline 1 & 205617.4 & +433818.8 & 0.015 & 48 & 18.12 & 1 \\
2 & 205707.1 & +434821.8 & 0.025 & 120 & 16.47 & 2 \\
3 & 205819.0 & +435332.5 & 0.023 & 74 & 4.93 & $3 \mathrm{~b}$ \\
4 & 205540.5 & +440620.0 & 0.025 & 39 & 5.68 & $\ldots$ \\
5 & 205054.9 & +442418.1 & 0.067 & 121 & 5.54 & 5 \\
6 & 205335.2 & +442757.4 & 0.075 & 130 & 6.27 & 6 \\
7 & 205914.2 & +441641.3 & 0.022 & 22 & 1.48 & $\ldots$ \\
8 & 204745.1 & +434329.4 & 0.017 & 28 & $\ldots$ & $\ldots$ \\
\hline
\end{tabular}

Note. The last column is the cluster label from Cambrésy et al. (2002), if appropriate.

Class II objects compared to the (presumably) younger Class I's is statistically lower than in the entire observed region by a factor of $\sim 1.3$. This is consistent with expectations-either due to high-velocity stars moving away from their birth sites, or as a result of the overall expansion of young clusters as they age due to, for example, removal of their remaining molecular cloud material by stellar winds. Moreover, the ratio $N$ (Class II)/ $N$ (Class II-III) decreases from denser to less dense regions, as expected for the same reasons, by a factor of $\sim 2$.

Figure 10 also shows the fractional number of background contaminants as compared to the fractional surface area inside each of the YSO density contours. The proportional number of background contaminants follows very closely the proportional surface area. This suggests that sources flagged as contaminants are really background objects and not YSOs; otherwise, one would expect an excess of sources flagged as background inside dense regions. 


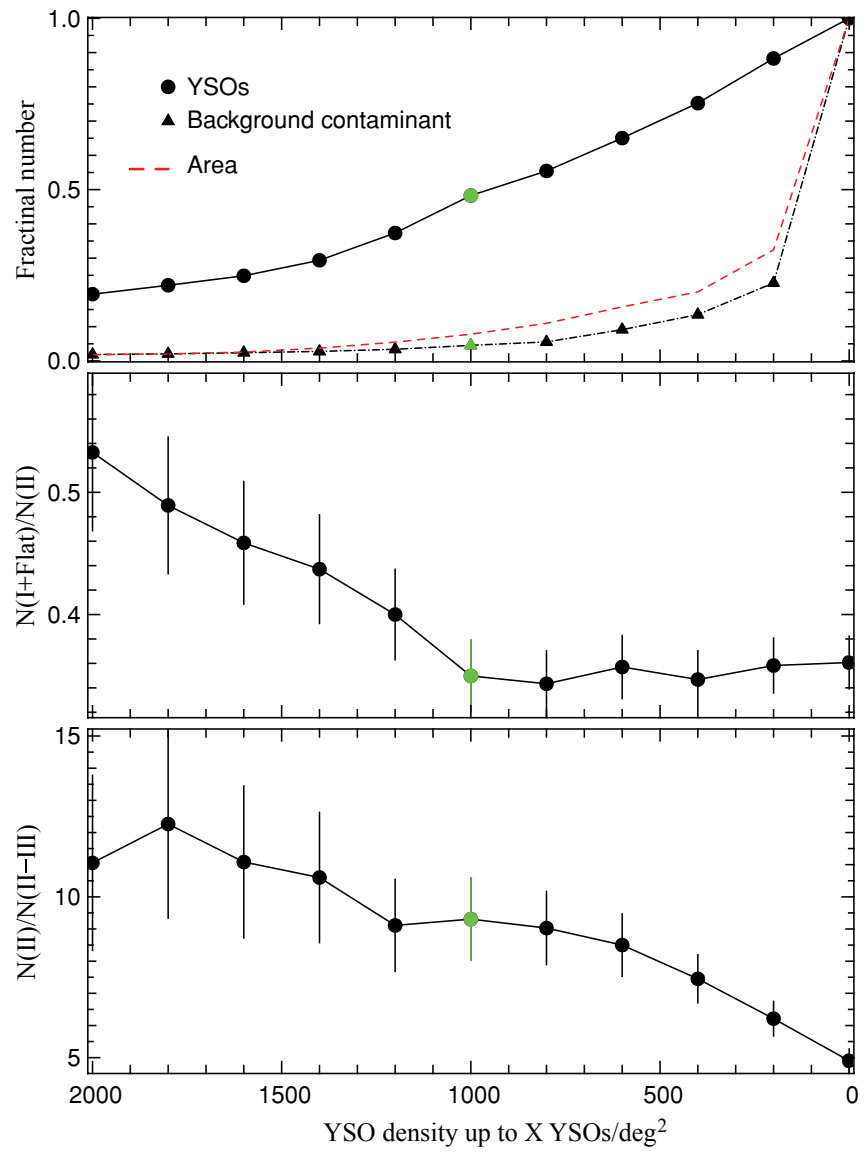

Figure 10. Ratios of object classes as a function of YSO density. The $x$-axis corresponds to the YSO density contours plotted in Figure 8 from high to low density (corresponding to small and large surface surrounded by each contour on Figure 8). Inside each contour, we calculated several fractional numbers. Top: the fraction of YSO candidates (filled dot symbols linked by solid line) and background contaminants (filled triangle liked by dot-dashed line); the red dashed line shows the surface area surrounded by each contour as a fraction of the total covered surface $\left(\sim 9 \mathrm{deg}^{2}\right)$. Middle: same, but for the fraction of (Class I+Flat)/Class II. Bottom: same, but for the fraction of Class II/Class II-III. Note that for the last value, $X=0$ corresponds physically to the boundary of our IRAC coverage.

(A color version of this figure is available in the online journal.)

Another way to characterize the degree of clustering, used by many authors, is the distribution of nearest neighbors. For each of our YSO candidates, we calculated the distance to the fourth nearest YSO candidates; Figure 11 shows the histogram of this distance, displayed for Class I+Flat, Class II, and Class II-III. We find a statistically significant difference between the three histograms: using a Kolmogorov-Smirnov test, the probability that each of these distributions is drawn from the same parent distribution as either of the other two distributions is less than $0.02 \%$. This is consistent with what we found above: the Class I population, usually assumed to be younger, is significantly more clustered than Class II-III

\section{HH OBJECTS}

Ogura et al. (2002) identified some $\mathrm{HH}$ objects using $\mathrm{H} \alpha$ over a small region of the complex near bright-rimmed cloud (BRC) 31. Bally \& Reipurth (2003) studied the entire Pelican Nebula using $\mathrm{H} \alpha,[\mathrm{N} I I]$, and [S II], finding several new $\mathrm{HH}$ objects.

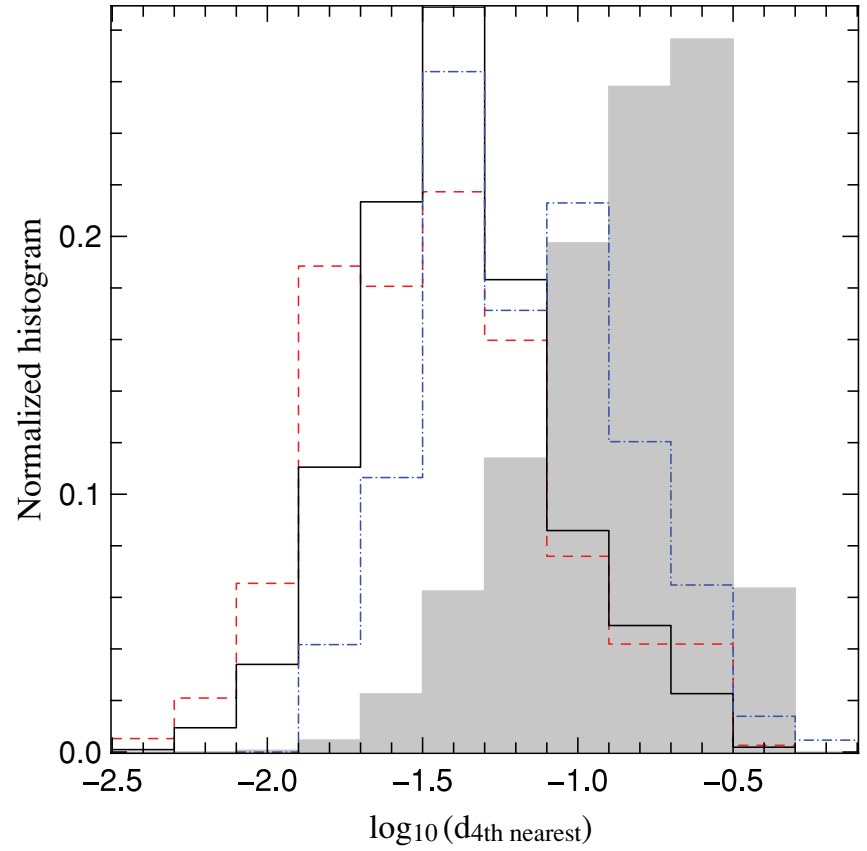

Figure 11. Histogram of the log of the distance of the fourth nearest YSO (in degrees) for each of our YSO candidates. The red dashed line is for Class I+Flat-spectrum sources, the solid black line for Class II stars, and the blue dashdotted histogram for Class II-III. The gray histogram represents the remaining population of noncluster members. The Class I population, usually assumed to be younger, is significantly more clustered than Class II-III; this is consistent with expectations that, as stars dynamically evolve, they move away from their birthplace.

(A color version of this figure is available in the online journal.)

The 3.5-8.5 $\mu \mathrm{m}$ wavelength range covered by the IRAC four channels is quite suitable to study deeply embedded young stellar outflows, since this range includes some of the brightest collisionally excited pure rotational $\mathrm{H}_{2}$ emission lines (Wright et al. 1996; Noriega-Crespo et al. 2004a, 2004b) as well as other $\mathrm{H}_{2}$ vibrational lines (Smith \& Rosen 2005). At the longer wavelengths, the MIPS channels sample a rich combination of atomic/ionic and molecular lines that include some of the best atomic species to study outflows, e.g., [Fe II] $25.98 \mu \mathrm{m}$, [O I] $63.18 \mu \mathrm{m}$, and [C II] $157.74 \mu \mathrm{m}$ (see, e.g., Molinari et al. 2000; Morris et al. 2004) that fall within the 24, 70, and $160 \mu \mathrm{m}$ bandpasses, respectively, plus of course continuum emission from cold dust. We discuss below our observations of one $\mathrm{HH}$ object discovered previously, HH 555.

HH 555 itself is not an embedded flow (like, e.g., HH 211) since it is clearly detected at optical wavelengths ( $\mathrm{H} \alpha$ and [S II] $6717 / 31 \AA$ ) and its spectrum is consistent with that of a shockexcited gas moving supersonically (Bally \& Reipurth 2003). HH 555 belongs to that special class of irradiated jets, like HH 399 in the Trifid nebula (Yusef-Zadeh et al. 2005) that are found in active star-forming regions and surrounded by a bath of ultraviolet (UV) ionizing photons from recently formed massive stars.

The case for HH 555 is particularly interesting because the jet and counter jet are bent westward as soon as they arise from their embedded source at the dense tip of the pillar, indicating the presence of stellar "sidewind" (Masciadri \& Raga 2001). The pillar itself is being photoevaporated leading to the formation of a two-shock structure (see Kajdič \& Raga 2007 for details). The length of time that the jet will maintain its bipolar morphology is expected to depend on the strength of the impinging ionizing flux. The two-shock structure developed by the interacting 


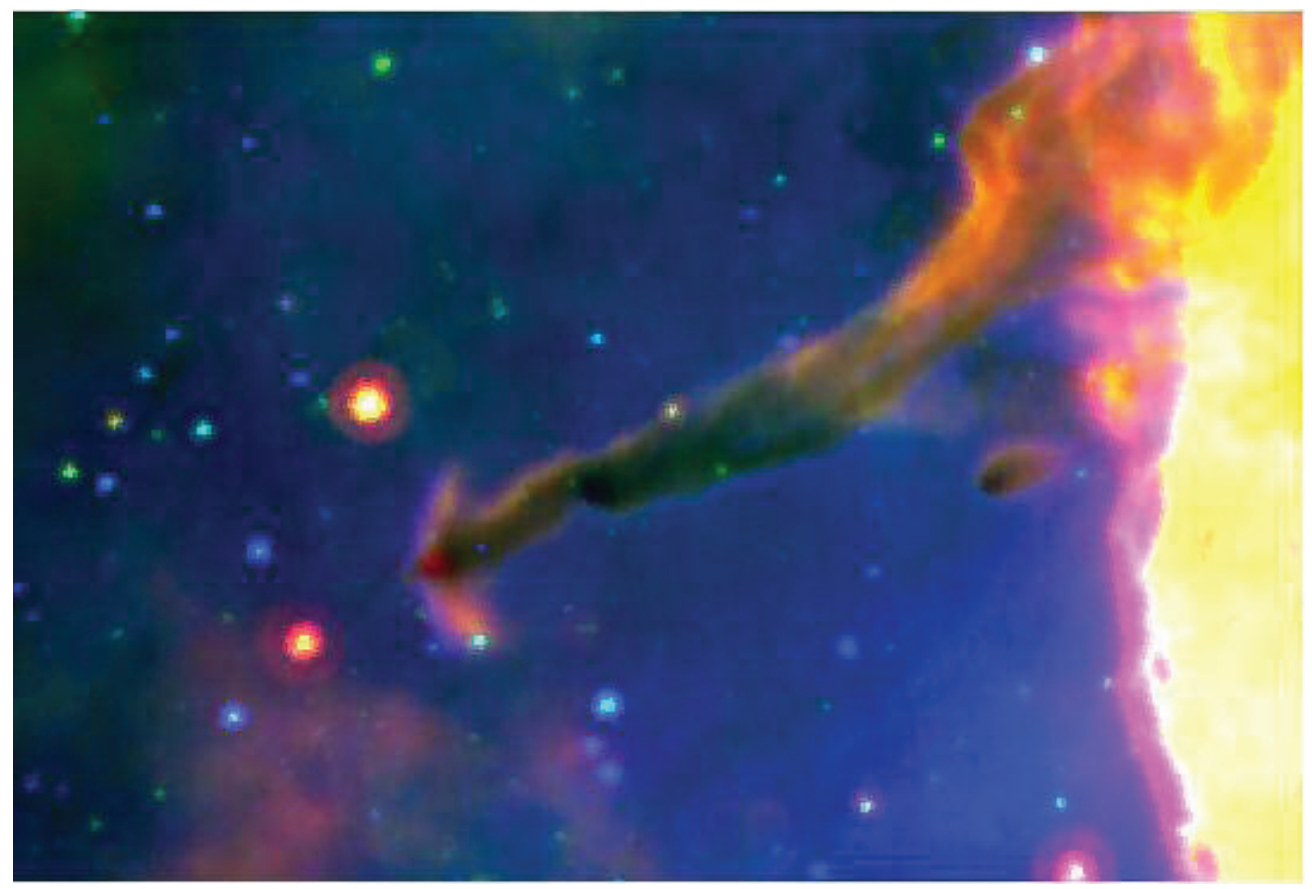

Figure 12. Three-color image of HH 555 arising from the tip of a dense pillar using H $\alpha$ (blue), IRAC $8 \mu \mathrm{m}$ (green), and MIPS $24 \mu \mathrm{m}$ (red). The FOV is $\sim 6^{\prime} \times 4^{\prime}$, and north is up and east is left. The mid-/far-IR bipolar jet ( $\sim 1^{\prime}$ in length) is centered on a $24 \mu \mathrm{m}$ embedded source. The stratification in ionization along the jet from east-to-west (the gradient in color) can be explained by the shield created during the two (stellar and photoevaporation) wind interaction (Kajdič \& Raga 2007).

Table 4

Table of YSO Candidates

\begin{tabular}{|c|c|c|c|c|c|c|c|c|c|c|c|c|c|c|}
\hline IAU Designation & R.A. (2000) & Decl. (2000) & 2MASS id & $B$ & $V$ & $I$ & $J$ & $H$ & $K_{s}$ & [3.6] & [4.5] & {$[5.8]$} & {$[8.0]$} & [24] \\
\hline SST205659.3+434753.0 & 205659.326 & 434752.993 & $20565934+4347530$ & 20.195 & 18.747 & 15.963 & 14.286 & 13.485 & 13.174 & 12.609 & 12.287 & 11.892 & 11.348 & \\
\hline SST205646.8+434609.0 & 205646.765 & 434608.980 & $20564675+4346089$ & 19.981 & 18.023 & 15.385 & 13.226 & 12.180 & 11.783 & 11.653 & 11.485 & 11.244 & 10.392 & \\
\hline
\end{tabular}

(This table is available in its entirety in a machine-readable form in the online journal. A portion is shown here for guidance regarding its form and content.)

winds actually creates a shield that protects both the jet and the pillar, and therefore, one would expect lower ionization on the west side of the outflow. Indeed, the Spitzer images combined with the ground-based $\mathrm{H} \alpha$ emission (Figure 12) confirm this stratification, if one assumes that the 8 and $24 \mu \mathrm{m}$ emission arises mostly from $\mathrm{H}_{2}$ and [S II], as is the case in other jets. This also explains why the $24 \mu \mathrm{m}$ source lies in the middle of the 8 and $24 \mu \mathrm{m}$ jets, but is slightly offset with respect to $\mathrm{H} \alpha$ (see, e.g., Kajdič \& Raga 2007, Figure 3).

Our $70 \mu \mathrm{m}$ images do not show a clear signature of the jet, and this could be due to a combination of things: lack of sensitivity, artifacts along the scanning direction and physics ([OI] $63.18 \mu \mathrm{m}$ is not present, neither is cold dust). Careful analyses of the data do show the presence of the source at $70 \mu \mathrm{m}$ at the tip of the pillar. The pillar itself, although faint, is distinguished and its emission is likely to be due to [O I] photodissociated emission. We measured the flux at 24 and $70 \mu \mathrm{m}$ using a small aperture of 7 and $16^{\prime \prime}$ respectively, centered at $20 \mathrm{~h} 51 \mathrm{~m} 19.52 \mathrm{~s}+44 \mathrm{~d} 25 \mathrm{~m} 38.3 \mathrm{~s}$. We found $10 \pm 3 \mathrm{mJy}$ at $24 \mu \mathrm{m}$ and $830 \pm 220 \mathrm{mJy}$ at $70 \mu \mathrm{m}$.

\section{CONCLUSION}

We have combined mid-IR photometry from Spitzer/IRAC with $J H K_{s}$ data from 2MASS to provide a sensitive photometric census of objects toward the North America and Pelican
Nebulae. We have used IRAC color and magnitude diagnostics to identify more than 1600 sources with IR excess characteristic of young stars surrounded by a disk.

Our YSO candidates (Table 4) are located primarily on the central dark cloud (Lynds 935). We identified eight main clusters, which together contain about a third of all the YSOs in the region; five of these clusters were previously recognized by Cambrésy et al. (2002). The sources we identify as background galaxies or AGN are relatively uniformly spread across the observed region. These two properties suggest a low level of contamination in our YSO sample.

We have assigned our YSOs classifications in the Class I/Flat/II/III system according to their IR SED slope. The proportion of Class I+Flat compared to Class II is variable within the NANeb, with the dark clouds called "Gulf of Mexico" having a remarkably higher proportion of Class I's, suggesting these clusters have a younger age than the rest of NANeb. The "Gulf of Mexico" region will be discussed in more detail in R09 where the MIPS data for NANeb will be presented.

We compared optical photometry of a small sample of the YSO candidates to evolutionary models in order to provide an age estimate for the NANeb. We found that most of these YSOs appear younger than $10 \mathrm{Myr}$ according to the isochrones of Siess et al. (2000), with the median age being 3 Myr. However, the most embedded and probably youngest YSOs 
were not included in this sample because our optical photometry is less sensitive than our IRAC observations and because the youngest stars are very red. However, despite all the caveats regarding this age estimation, it is the most robust published so far.

Finally, thanks to our IR images from 3.6 to $70 \mu \mathrm{m}$, we have provided new clues on the nature of $\mathrm{HH} 555$, a peculiar $\mathrm{HH}$ object reported in Bally \& Reipurth (2003). We detected a source at $70 \mu \mathrm{m}$ at the base of the bi-polar jets. Our data add support to the hypothesis that the bent shape of HH 555 is a result of having the jet propagate in an environment with a stellar "sidewind."

This work is based, in part, on observations made with the Spitzer Space Telescope, which is operated by the Jet Propulsion Laboratory, California Institute of Technology under a contract with NASA. Support for this work was provided by NASA through an award issued by JPL/Caltech. This research has made use of NASA's Astrophysics Data System (ADS) Abstract Service, and the SIMBAD database, operated at CDS, Strasbourg, France. This research has made use of data products from the Two Micron All Sky Survey (2MASS), which is a joint project of the University of Massachusetts and the Infrared Processing and Analysis Center, funded by the National Aeronautics and Space Administration and the National Science Foundation. These data were served by the NASA/ IPAC Infrared Science Archive, which is operated by the Jet Propulsion Laboratory, California Institute of Technology, under contract with the National Aeronautics and Space Administration. This research has made use of the Digitized Sky Surveys, which were produced at the Space Telescope Science Institute under US Government grant NAG W-2166. The images of these surveys are based on photographic data obtained using the Oschin Schmidt Telescope on Palomar Mountain and the UK Schmidt Telescope. The plates were processed into the present compressed digital form with the permission of these institutions.

The research described in this paper was partially carried out at the Jet Propulsion Laboratory, California Institute of Technology, under contract with the National Aeronautics and Space Administration.

\section{REFERENCES}

André, P. 1994, in The Cold Universe, ed. T. Montmerle, et al. (France: Editions Frontieres), 179

Armond, A. C., Reipurth, B., \& Vaz, L. P. 2003, Bull. Astron. Soc. Brazil, 23, 92

Bally, J., \& Reipurth, B. 2003, AJ, 126, 893

Bally, J., \& Scoville, N. Z. 1980, ApJ, 239, 121

Baraffe, I., Chabrier, G., Allard, F., \& Hauschildt, P. H. 1998, A\&A, 337, 403

Cambrésy, L., Beichman, C. A., Jarrett, T. H., \& Cutri, R. M. 2002, AJ, 123 2559

Comerón, F., \& Pasquali, A. 2005, A\&A, 430, 541

Fazio, G. G., et al. 2004, ApJS, 154, 10

Flaherty, K. M., et al. 2007, ApJ, 663, 1069

Gutermuth, R. A., et al. 2008, ApJ, 674, 336

Harvey, P. M., et al. 2006, ApJ, 644, 307

Harvey, P., et al. 2007a, ApJ, 663, 1149

Harvey, P. M., et al. 2007b, ApJ, 663, 1139

Herbig, G. H. 1958, ApJ, 128, 259

Jeffries, R. D., Oliveira, J. M., Naylor, T., Mayne, N. J., \& Littlefair, S. P. 2007, MNRAS, 376, 580

Jørgensen, J. K., Johnstone, D., Kirk, H., Myers, P. C., Allen, L. E., \& Shirley, Y. L. 2008, ApJ, 683, 822

Kajdič, P., \& Raga, A. C. 2007, ApJ, 670, 1173

Lada, C. J. 1987, in IAU Symp. 115, Star Forming Regions, ed. M. Peimbert \& J. Jugaku (Dordrecht: Kluwer), 1

Laugalys, V., \& Straižys, V. 2002, Balt. Astron., 11, 205

Luhman, K. L., et al. 2003, ApJ, 593, 1093

Makovoz, D., \& Marleau, F. R. 2005, PASP, 117, 1113

Masciadri, E., \& Raga, A. C. 2001, AJ, 121, 408

Molinari, S., et al. 2000, ApJ, 538, 698

Morris, P. W., et al. 2004, ApJS, 154, 339

Muench, A. A., Lada, C. J., Luhman, K. L., Muzerolle, J., \& Young, E. 2007, AJ, 134,411

Noriega-Crespo, A., et al. 2004a, ApJS, 154, 402

Noriega-Crespo, A., et al. 2004b, ApJS, 154, 352

Ogura, K., Sugitani, K., \& Pickles, A. 2002, AJ, 123, 2597

Rebull, L. M., et al. 2002, AJ, 123, 1528

Rebull, L. M., et al. 2009, AAS, 213, 413.13

Siess, L., Dufour, E., \& Forestini, M. 2000, A\&A, 358, 593

Silverman, B. W. 1986, Density Estimation for Statistics and Data Analysis, (Monographs on Statistics and Applied Probability; London: Chapman and Hall)

Skrutskie, M. F., et al. 2006, AJ, 131, 1163

Smith, M. D., \& Rosen, A. 2005, MNRAS, 357, 1370

Stauffer, J. R. 1996, in ASP Conf. Ser. 109, Cool Stars, Stellar Systems, and the Sun, ed. R. Pallavicini \& A. K. Dupree (San Francisco, CA: ASP), 305

Werner, M. W., et al. 2004, ApJS, 154, 1

Winston, E., et al. 2007, ApJ, 669, 493

Wright, C. M., et al. 1996, A\&A, 315, L301

Yusef-Zadeh, F., Biretta, J., \& Wardle, M. 2005, ApJ, 624, 246 